\title{
基于苯霜灵代谢物结构的双酰胺化合物的合成与杀菌活性研究
}

\author{
孙国绍王玲娜金淑惠* 董燕红路慧哲张建军* \\ (中国农业大学理学院 北京 100193)
}

\begin{abstract}
摘要 基于苯霜灵代谢物结构骨架，采用活性基团拼接法，以 $N^{2}$-(丙二酸单酰基)- $N^{1}$-(2,6-二甲苯基)丙氨酸甲酯为母体, 设计合成了 14 个结构新颖的双酰胺类化合物, 通过 ${ }^{1} \mathrm{H} N M R,{ }^{13} \mathrm{C} N M R$ 及高分辨质谱进行结构确证. 生测结果表明, 目 标化合物均有一定的杀菌活性, 部分化合物的杀菌活性优于苯霜灵. 在 $50 \mathrm{mg} / \mathrm{L}$ 浓度下, 化合物 7d 对水稻纹枯病原菌 的抑制率为 $94.2 \%$, 化合物 $7 \mathbf{k}$ 对辣椒疫霉和棉花立枯病原菌的抑制率分别为 $87.1 \%$ 和 $88.9 \%$.

关键词 苯霜灵; 代谢物; 双酰胺; 合成; 杀菌活性
\end{abstract}

\section{Synthesis and Fungicidal Activity of Diamide Compounds Based on the Metabolite of Benalaxyl}

\author{
Sun, Guoshao Wang, Lingna Jin, Shuhui* Dong, Yanhong \\ Lu, Huizhe Zhang, Jianjun* \\ (China Agricultural University, Beijing 100193)
}

\begin{abstract}
Based on benalaxyl pesticide metabolite structure framework, fourteen novel diamide derivatives were synthesized by the reaction of amine and $N^{2}$-carboxyacetyl- $N^{1}$-(2,6-dimethylphenyl)alanine methyl ester. Their structures were identified by ${ }^{1} \mathrm{H}$ NMR, ${ }^{13} \mathrm{C}$ NMR spectra and HRMS. The results of bioassay indicated that some compounds possess potential fungicidal activities against 8 kinds of bacteria, and the antibacterial activity of some compounds was better than that of benalaxyl. At a dosage of $5.0 \times 10^{-5} \mathrm{~g} / \mathrm{mL}$, compound $\mathbf{7 d}$ displayed $94.2 \%$ antifungal activities against Rhizoctonia solani. Compound $\mathbf{8 f}$ displayed $87.1 \%$ and $88.9 \%$ antifungal activities against Phytophthora capsici and Rhizoctonia solani.

Keywords benalaxyl; metabolite; diamide; synthesis; antifungal activity
\end{abstract}

20 世纪 30 年代, 德国科学家偶然发现百浪多息在 试管内无杀菌活性，在生物体内却可以杀死细菌这一反 常现象，后研究证明百浪多息中的有效成分是其代谢产 物对氨苯磺胺, 进而发现了磺胺类药物 ${ }^{[1]}$. 1940 年, Wood 和 Fileds 建立了代谢拮抗学说, 新颖的代谢物结 构成为医药先导化合物的发现方法之一, 被广泛应用于 医药行业, 如由阿司咪唑进一步发现诺阿司咪唑, 提高 了对靶标受体的选择性, 增加了安全性 ${ }^{[2]}$.

有机农药作为外源化学物质进入环境之中, 不可避 免地在环境的各组成要素之中迁移并被转化, 导致产生 大量代谢产物, 相对于母体, 代谢产物往往会降低或丧 失母体的活性, 但是一些代谢产物的活性比母体进一步 提高: 马拉硫磷的代谢物马拉氧磷对乙酰胆碱酯酶的抑
制活性提高约 2 个数量级 ${ }^{[3]}$, 噁唑禾草灵的代谢产物噁 唑禾草灵酸对脂肪酸重新合成的抑制率比母体高 10 倍 ${ }^{[4]}$, 噻虫嗪代谢产物噻虫胺比母体具有更好的杀虫活 性 ${ }^{[5,6]}$ (图 1), 可见，农药的代谢产物化学结构也可为新 农药创制先导化合物的发现提供重要的启示.

酰胺类化合物是医药、农药和兽药等研究领域中广 受关注的化学结构, 往往具有独特的杀虫、杀菌、除草 以及植物生长调节等活性 ${ }^{[7 ~ 15]}$, 如苯霜灵、甲霜灵、呋 霜灵等均是成功的商品化酰胺类杀菌剂品种(图 2), 其 化学结构中都含有 $N$-(2,6-二甲基苯基)丙酸甲酯基团, 可见该结构对杀菌活性的发挥具有十分重要的作用. 苯 霜灵是一种高效、低毒、持效期长的内吸性杀菌剂，能 够抑制核糖体 RNA 的合成，导致蛋白质合成减少，从

* Corresponding author. E-mail: shuhuij@cau.edu.cn; zhangjianjun@cau.edu.cn

Received August 10, 2016; revised August 31, 2016; published online September 8, 2016.

Project supported by National Science Foundation for Fostering Talents in Basic Research of China (No. J1210064) and the National Natural Science Fondation of China (No. 21337005).

国家基础科学人才培养基金(No. J1210064)及国家自然科学基金(No. 21337005)资助项目. 
而抑制菌丝体的生长，被广泛应用于防治霜霉病、早疫 病和晚疫病等卵菌纲病害 ${ }^{[16,17]}$. 苯霜灵的代谢物主要有 三个，代谢物 $\mathrm{A} 、$ 代谢物 $\mathrm{B}$ 和代谢物 $\mathrm{C}^{[18,19]}$ (图 2), 其中 代谢物 $\mathrm{A}$ 保留了母体中的 $N-(2,6-$ 二甲基苯基)丙酸甲酯 基药效团，在国家自然基金重点项目 “农药代谢物在水 生环境及水生生物中的生成机制及健康效应研究” 研究 中, 课题组前期合成了苯霜灵的三个典型代谢物的标 样, 生物活性篎选表明, 代谢物 $\mathrm{A}$ 其具有较好的杀菌活
性，其对辣椒疫霉和番茄晚疫病原菌的抑制率分别为 $50.3 \%$ 和 $70.2 \%$ ，因此本文以苯霜灵代谢物 A 为先导，通 过结构优化设计合成系列未见文献报道的双酰胺类化 合物(图 2), 其结构经过核磁氢谱、碳谱以及高分辨质谱 的确认，初步生物活性测试结果表明，在 $50 \mathrm{mg} / \mathrm{L}$ 浓度 下，目标化合物 7d, 7e 和 7k 有较好的杀菌活性，目标化 合物合成路线如 Scheme 1 所示.<smiles>CCOC(=O)CC(SP(=S)(OC)OC)SP(=S)(OC)OC(C)C(=O)OCC</smiles><smiles></smiles><smiles>CN/C(=N/O[N+](=O)[O-])NCc1cnc(Cl)s1</smiles>

噻虫嗪<smiles>CC(Oc1ccc(Oc2nc3ccc(Cl)cc3o2)cc1)C(=O)O</smiles>

图 1 农药及其代谢产物

Figure 1 Pesticides and their metabolites
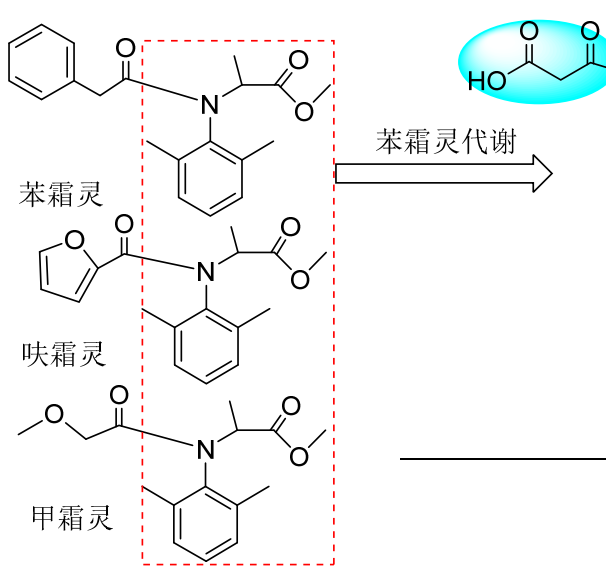<smiles>Cc1cccc(C)c1N(C)C(C)C(=O)O</smiles>

代谢物A<smiles>CC(=O)OCc1cccc(C)c1N(C(=O)Cc1ccccc1)C(C)(C)C</smiles>

代谢物B<smiles>Cc1cccc(C)c1N(C(=O)Cc1ccccc1)C(C)C(=O)O</smiles>

代谢物C

图 2 设计目标化合物

Figure 2 Target compound design
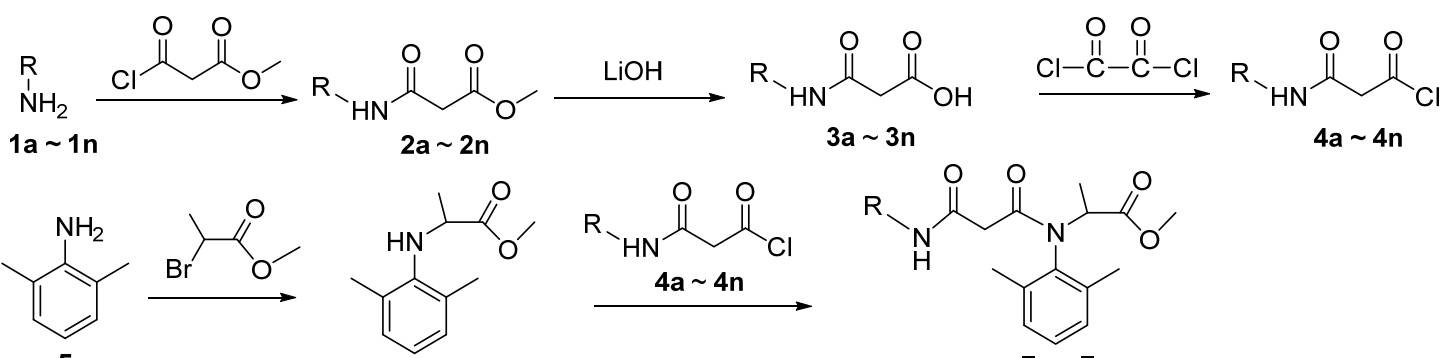<smiles>COC(=O)C(C)Nc1c(C)cccc1C</smiles><smiles>[R]NC(=O)CC(C)CC(=O)Cl</smiles><smiles>[R]NC(=O)CC(=O)N(c1c(C)cccc1C)C(C)C(=O)OC</smiles>

7a: $\mathrm{R}=2,5-\left(\mathrm{CF}_{3}\right)_{2}-\mathrm{C}_{6} \mathrm{H}_{3} ; \mathbf{7 b}: \mathrm{R}=2-\mathrm{Cl}_{-}-\mathrm{CF}_{3}-\mathrm{C}_{6} \mathrm{H}_{3} ; \mathbf{7 c}: \mathrm{R}=\mathrm{C}_{6} \mathrm{H}_{5} ; \mathbf{7 d}: \mathrm{R}=2-\left(4-\mathrm{Cl}_{-} \mathrm{C}_{6} \mathrm{H}_{5}\right)-\mathrm{C}_{6} \mathrm{H}_{4} ; 7 \mathbf{7 e}: \mathrm{R}=3-\mathrm{CF}_{3}-\mathrm{C}_{6} \mathrm{H}_{4} ; \mathbf{7 f}: \mathrm{R}=2-\mathrm{CN}-\mathrm{C}_{6} \mathrm{H}_{4} ; 7 \mathbf{7 g}: \mathrm{R}=$ 4- $\mathrm{C}_{2} \mathrm{H}_{5} \mathrm{O}-\mathrm{C}_{6} \mathrm{H}_{4} ; 7 \mathbf{7 h}: \mathrm{R}=3-\mathrm{CH}_{3} \mathrm{O}-\mathrm{C}_{6} \mathrm{H}_{4} ; 7 \mathrm{i}: \mathrm{R}=2-\mathrm{CH}_{3} \mathrm{O}-\mathrm{C}_{6} \mathrm{H}_{4} ; 7 \mathbf{7 j}: \mathrm{R}=4-\mathrm{Me}_{-} \mathrm{C}_{6} \mathrm{H}_{4} ; \mathbf{7 k}: \mathrm{R}=4-\mathrm{Cl}-\mathrm{C}_{6} \mathrm{H}_{4} ; 7 \mathrm{l}: \mathrm{R}=2-\mathrm{Me}-5-\mathrm{F}-\mathrm{C}_{6} \mathrm{H}_{3} ; \mathbf{7 m}: \mathrm{R}=$ 4-Me-3-Cl- $\mathrm{C}_{6} \mathrm{H}_{3} ; \mathbf{7 n}: \mathrm{R}=3-\mathrm{CF}_{3}-4-\mathrm{Br}-\mathrm{C}_{6} \mathrm{H}_{3}$

图式 1 目标化合物合成路线

Scheme 1 Synthetic route of target compound 


\section{1 结果与讨论}

\section{1 合成讨论及波谱分析}

目标化合物的合成方法主要有两种: (1)丙二酰氯选 择性地分别和化合物 1、中间体 6 反应; (2)通过 Scheme 3 所示方法合成目标化合物. 第一种方法反应过程不易 跟踪监测，易产生丙二酰氯与化合物 $\mathbf{1}$ 或中间体 $\mathbf{6}$ 的双 取代产物, 难以与目标化合物 7 分离, 最后得到的产物 成分复杂, 纯度差, 因此我们采用第二种方法合成目标 化合物.

中间体 2 的合成方法主要有两种: (1)丙二酸单甲酯, DCC 缩合; (2)丙二酸单甲酯酰氯, 三乙胺作缚酸剂进行 酰胺化. 第一种方法反应时间长, DCC 较难除净, 给后 处理带来很大不便, 得到的中间体 2 纯度较低. 第二种 方法在冰浴条件下, 将丙二酸单甲酯酰氯滴加到化合物 $\mathbf{1}$ 的二氯甲烷溶液中, $0.5 \mathrm{~h}$ 即可反应完毕, 重结晶便可 得到高纯度的中间体 2 , 因此选择第二种方法合成中间 体 2.

中间体 3 合成过程中, 选择由氢氧化钠水解中间体 2 , 发现酯键和酰胺键均断裂, 产物很杂. 选择由碱性较 弱的氢氧化锂水解, 可以选择性地脱去酯基, 而不影响 酰胺键，得到高纯度中间体 $\mathbf{3}$.

合成中间体 4 的方法主要有两种: (1)中间体 3 和二 氯亚砜回流制得; (2)中间体 3 和草酰氯反应制得. 第一 种方法中氯化亚砜有强烈的刺激性气味, 并且毒性较 大, 反应条件需要高温回流, 而第二种方法仅需要在冰 浴条件下滴加草酰氯, 并加入催化量的 DMF, 约 $2 \sim 3 \mathrm{~h}$ 便可反应完全, 因此我们选择第二种方法来制得中间体 4.

合成中间体 6 时, 反应温度控制在 $125{ }^{\circ} \mathrm{C}$ 左右, 低 温造成反应时间延长, 高温则会使 2-澳丙酸甲酯回流, 影响反应的进行，高温低温均会使产率降低.

合成目标化合物 7 时, 尝试了不同反应条件对收率 的影响. 当选用三乙胺作缚酸剂、二氯甲烷作溶剂时, 和吡啶作溶剂和缚酸剂时, 反应 $5 \mathrm{~h}$ 并尝试了加热回流 反应, 仍只有很少产物产生. 选用甲苯作溶剂、碳酸氢 钠作缚酸剂时, 室温反应 $4 \sim 5 \mathrm{~h}$, 基本反应完全. 所以 最终选用该方法制备目标化合物 7.

以化合物 7c 为例, 进行核磁氢谱和碳谱分析. $\mathrm{CONH}$ 由于受到羰基和苯环的吸电子作用, 氢原子共振 峰移向低场, $\delta$ 值为 9.88, 呈单峰. 苯环上的氢的 $\delta$ 值在 $7.00 \sim 7.60$ 左右, 且为多重峰, 位置较难归属. $\mathrm{COCH}_{2} \mathrm{CO}$ 中氢受到两个羰基的吸电子作用, $\delta$ 值为 3.08 , 并且两个氢之间自偶合, 形成 $\mathrm{dd}$ 峰, 偶合常数为 17.7 Hz. $\delta$ 在 $172.5,170.1,163.6$ 处的峰为三个羰基碳原 子的信号峰.

\section{2 杀菌活性}

将目标化合物配制成浓度为 $5.0 \times 10^{-} 5 \mathrm{~g} / \mathrm{mL}$ 药液, 采用菌体生长速率法 ${ }^{[20]}$ 对辣椒疫霉病菌 (Phytophthora capsici)、番茄早疫病菌(Alternaria solani)、水稻纹枯病 菌(Rhizoctonia solani)、油菜菌核病菌(Sclerotinia sclerotiorum)、番茄晚疫病菌(Phtophthora infestans)棉花立 枯病菌(Rhizoctonia solani Kuhn)、番茄灰霉病菌(Botrytis cinerea Pers)、棉花枯萎病菌(Fsarium oxysporum) 等 8 种 菌进行了初步杀菌活性测试, 化合物杀菌普筛结果见表 1.

由表 1 可知, 目标化合物对 8 种菌均表现出一定的 抑制活性, 部分化合物的抑菌活性优于苯霜灵. 对棉花 枯萎、油菜菌核、番茄晚疫和番茄早疫病原菌活性较差, 只有少数目标化合物表现出 $70 \%$ 以上的抑菌活性. 对水 稻纹枯、棉花立枯、辣椒疫霉和番茄灰霉病原菌表现出 良好抑菌活性, 其中, 化合物 $7 \mathbf{k}$ 对辣椒疫霉病原菌的抑 菌活性达到 87\%, 优于苯霜灵; 化合物 7d 对水稻纹枯和 棉花立枯病原菌的抑制率分别为 $94.2 \%$ 和 $85.9 \%$, 化合 物 $7 \mathbf{k}$ 对水稻纹枯和棉花立枯病原菌的抑制率分别为 $80.6 \%$ 和 $88.9 \%$, 化合物 $7 \mathbf{j}$ 对番茄灰霉病原菌的抑制率 为 $86.2 \%$. 总体来说, 当取代基为吸电子基时, 化合物 表现出更好的抑菌活性, 如化合物 7d, 7k 对大多数病原 菌表现出良好的抑菌活性, 化合物 71 和 $7 \mathrm{~m}$ 总体抑菌活 性优于化合物 $7 \mathrm{j}$; 单吸电子基取代的化合物比双吸电子 基取代的化合物具有更好的抑菌活性，比如，化合物 7e 抑菌活性优于化合物 $7 \mathbf{a}$ 和 $\mathbf{7 n}$.

\section{2 结论}

本文以苯霜灵代谢物 $N^{2}$-(丙二酸单酰基)- $N^{1}$-(2,6-二 甲苯基)丙氨酸甲酯结构为先导, 设计合成了 14 个结构 新䓉的双酰胺类化合物, 并对其进行了杀菌活性测试. 生测结果表明, 目标化合物均有一定的杀菌活性, 其中, 化合物 7d 对番茄早疫、水稻纹枯和棉花立枯病原菌的 抑制率分别为 $84.3 \%, 94.2 \%$ 和 $85.9 \%$, 均优于苯霜灵. 苯环上 $\mathrm{R}$ 基团吸/推电子的性质和位置的不同，对目标 化合物杀菌活性有一定影响, 通过对目标化合物结构和 抑菌活性之间的关系分析, 当取代基为单取代吸电子基 时, 目标化合物的杀菌活性更好, 如化合物 7d, 7e, 7k均 表现出良好的离体杀菌活性, 其活体杀菌活性研究正在 开展之中.

\section{3 实验部分}

\section{1 仪器与试剂}

质谱采用 Bruker Daltonics Bio-TOF-Q III 型质谱仪 (ESIMS)测定; 核磁共振波谱使用 Bruker Avance 300 
表 1 目标化合物的抑菌活性数据 $\left(5.0 \times 10^{-} 5 \mathrm{~g} / \mathrm{mL}\right)$

Table 1 Fungicidal activity of target compounds $\left(5.0 \times 10^{-} 5 \mathrm{~g} / \mathrm{mL}\right)$

\begin{tabular}{|c|c|c|c|c|c|c|c|c|}
\hline 化合物 & 辣椒疫霉 & 番茄晚疫 & 水稻纹枯 & 棉花立枯 & 棉花枯萎 & 油菜菌核 & 番茄灰霉 & 番茄早疫 \\
\hline $7 a$ & 55.6 & 83.0 & 31.7 & 60.9 & 31.6 & 57.4 & 62.4 & 30.1 \\
\hline $7 b$ & 72.0 & 58.1 & 51.9 & 66.0 & 34.8 & 51.6 & 81.0 & 53.9 \\
\hline $7 c$ & 61.4 & 76.0 & 15.4 & 66.0 & 34.8 & 36.7 & 57.9 & 34.2 \\
\hline $7 d$ & 76.0 & 63.1 & 94.2 & 85.9 & 25.1 & 57.4 & 68.1 & 84.3 \\
\hline $7 e$ & 74.0 & 63.1 & 81.8 & 72.9 & 43.8 & 74.0 & 75.0 & 56.4 \\
\hline $7 f$ & 45.4 & 83.0 & 46.2 & 52.7 & 57.2 & 46.0 & 56.3 & 33.3 \\
\hline $7 \mathrm{~g}$ & 76.0 & 41.5 & 51.9 & 56.6 & 25.1 & 30.0 & 64.4 & 48.6 \\
\hline $7 \mathrm{~h}$ & 29.9 & 44.5 & 40.2 & 66.0 & 40.8 & 33.3 & 62.4 & 24.7 \\
\hline $7 \mathbf{i}$ & 41.5 & 38.3 & 31.7 & 63.5 & 28.4 & 26.7 & 71.2 & 27.9 \\
\hline $7 \mathbf{j}$ & 71.3 & 63.1 & 50.1 & 56.6 & 21.7 & 57.4 & 86.2 & 48.6 \\
\hline $7 k$ & 87.1 & 71.2 & 80.6 & 88.9 & 21.7 & 74.0 & 68.1 & 58.8 \\
\hline 71 & 74.6 & 60.6 & 67.1 & 77.0 & 31.6 & 62.5 & 82.4 & 48.6 \\
\hline $7 m$ & 49.3 & 38.5 & 78.2 & 75.0 & 21.7 & 62.5 & 66.3 & 61.2 \\
\hline $7 n$ & 43. & 28.9 & 59.0 & 72.9 & 18.3 & 33.3 & 58.4 & 48.6 \\
\hline 苯霜灵 & 74.6 & 90.2 & 74.0 & 60.7 & 80.9 & 40.8 & 36.7 & 75.0 \\
\hline 代谢物 A & 50.3 & 70.2 & 12.9 & 37.3 & 31.6 & 33.3 & 66.3 & 11.0 \\
\hline
\end{tabular}

型核磁共振仪测定, 四甲基硅烷(TMS)为内标. 所有试 剂为国产市售分析纯，无水溶剂按标准方法处理.

\section{2 实验方法}

\subsection{1中间体 2 的合成}

中间体 2a $\sim 2 \mathbf{n}$ 的制备参考文献[21]. 在 $250 \mathrm{~mL}$ 的 圆底烧瓶中加入化合物 1 (20 mmol), 无水二氯甲烷 30 $\mathrm{mL}$ 溶解, 在冰浴和氮气保护条件下, 加入三乙胺 $(5.06$ $\mathrm{g}, 50 \mathrm{mmol}), 5 \mathrm{~min}$ 后缓慢滴加丙二酸单甲酯酰氯二氯 甲烷溶液 $15 \mathrm{~mL}$ (3.28 g, $24 \mathrm{mmol})$, 滴完后继续反应约 3 $\mathrm{h}$, 薄层色谱(TLC)监测反应完全, 甲醇淬灭反应. 依次 用 $1 \mathrm{~mol} / \mathrm{L}$ 盐酸、饱和氯化钠溶液、水洗涤有机相, 无 水硫酸钠干燥后蒸去二氯甲烷, 柱层析纯化得到中间体 2.

3-羰基-3-(2,5-二三氟甲基苯胺基)丙酸甲酯(2a): 白 色固体, 产率 94\%. m.p. 101 102 ${ }^{\circ} \mathrm{C} ;{ }^{1} \mathrm{H}$ NMR (300 $\mathrm{MHz}, \mathrm{CDCl}_{3}$ ) $\delta: 9.89$ (s, 1H, CONH), 8.65 (s, 1H, ArH), 7.77 (d, $J=8.2 \mathrm{~Hz}, 1 \mathrm{H}, \mathrm{ArH}), 7.50$ (d, $J=8.2 \mathrm{~Hz}, 1 \mathrm{H}$, $\mathrm{ArH}), 3.84$ (s, 3H, $\left.\mathrm{OCH}_{3}\right), 3.57$ (s, $\left.2 \mathrm{H}, \mathrm{COCH}_{2} \mathrm{CO}\right) ;{ }^{13} \mathrm{C}$ NMR $\left(75 \mathrm{MHz}, \mathrm{CDCl}_{3}\right) \delta: 169.34,163.29,135.40,134.35$ $(\mathrm{q}, J=33.3 \mathrm{~Hz}), 126.45(\mathrm{q}, J=5.4 \mathrm{~Hz}), 124.57$ (q, $J=$ $271.6 \mathrm{~Hz}), 124.52$ (q, $J=272.0 \mathrm{~Hz}), 122.89$ (q, $J=30.7$ $\mathrm{Hz}$ ), 120.83 (q, $J=5.3 \mathrm{~Hz}$ ), 52.27, 40.66; HRMS (ESI) calcd for $\mathrm{C}_{11} \mathrm{H}_{8} \mathrm{~F}_{6} \mathrm{NO}_{3}[\mathrm{M}+\mathrm{H}]^{+}:$316.0403, found 316.0399 .

3-羰基-3-(2-氯-4-三氟甲基苯胺基)丙酸甲酯(2b): 白色固体, 产率 92\%. m.p. 73 74 ${ }^{\circ} \mathrm{C} ;{ }^{1} \mathrm{H}$ NMR (300 $\left.\mathrm{MHz} \mathrm{CDCl}_{3}\right) \delta: 10.03$ (s, 1H, CONH), 8.56 (d, $J=8.7 \mathrm{~Hz}$, 1H, ArH), 7.64 (s, 1H, ArH), 7.51 (d, $J=8.7 \mathrm{~Hz}, 1 \mathrm{H}, \mathrm{ArH})$, 3.84 (s, $3 \mathrm{H}, \mathrm{O} \mathrm{CH}$ ), 3.58 (s, 2H, $\left.\mathrm{COCH}_{2} \mathrm{CO}\right) ;{ }^{13} \mathrm{C} \mathrm{NMR}$ $\left(75 \mathrm{MHz}, \mathrm{CDCl}_{3}\right) \delta: 169.75,163.11,137.41,126.44$ (q, $J=33.4 \mathrm{~Hz}$ ), 126.08 (q, $J=3.9 \mathrm{~Hz}), 124.55$ (q, $J=3.7 \mathrm{~Hz}$ ), 123.08 (q, $J=270.3 \mathrm{~Hz}$ ), 122.95, 121.08, 52.61, 41.15; HRMS (ESI) calcd for $\mathrm{C}_{11} \mathrm{H}_{10} \mathrm{ClF}_{3} \mathrm{NO}_{3}[\mathrm{M}+\mathrm{H}]^{+}$: 296.0296, found 296.0293.

3-羰基-3-(苯胺基)丙酸甲酯(2c) ${ }^{[22]}$ : 棕色固体，产 率 96\%. m.p. 43 44 ${ }^{\circ} \mathrm{C}$ (文献值 $42 \sim 44{ }^{\circ} \mathrm{C}$ ); ${ }^{1} \mathrm{H} \mathrm{NMR}$ $\left(300 \mathrm{MHz}, \mathrm{CDCl}_{3}\right) \delta: 9.34(\mathrm{~s}, 1 \mathrm{H}, \mathrm{CONH}), 7.61 \sim 7.47(\mathrm{~m}$, $2 \mathrm{H}, \mathrm{ArH}), 7.33 \sim 7.18(\mathrm{~m}, 2 \mathrm{H}, \mathrm{ArH}), 7.12 \sim 6.95(\mathrm{~m}, 1 \mathrm{H}$, $\mathrm{ArH}), 3.67$ (s, 3H, $\left.\mathrm{OCH}_{3}\right), 3.45$ (s, 2H, $\left.\mathrm{COCH}_{2} \mathrm{CO}\right)$.

3-羰基-3-(4-氯联苯-2-胺基)丙酸甲酯(2d): 淡黄色 固体, 产率 83\%. m.p. 87 $88{ }^{\circ} \mathrm{C}$; ${ }^{1} \mathrm{H}$ NMR (300 MHz, $\left.\mathrm{CDCl}_{3}\right) \delta: 8.74(\mathrm{~s}, 1 \mathrm{H}, \mathrm{CONH}), 8.20(\mathrm{~d}, J=8.2 \mathrm{~Hz}, 1 \mathrm{H}$, $\mathrm{ArH}), 7.68 \sim 7.11(\mathrm{~m}, 7 \mathrm{H}, \mathrm{ArH}), 3.67\left(\mathrm{~s}, 3 \mathrm{H}, \mathrm{OCH}_{3}\right), 3.38$ (s, 2H, $\left.\mathrm{COCH}_{2} \mathrm{C} \mathrm{O}\right) ;{ }^{13} \mathrm{C} \mathrm{NMR}\left(75 \mathrm{MHz}, \mathrm{CDCl}_{3}\right) \delta$ : $169.04,162.82,136.43,134.21,133.76,132.20,130.56$, $129.95,128.83,128.44,124.85,122.44,52.30,41.73$; HRMS (ESI) calcd for $\mathrm{C}_{16} \mathrm{H}_{15} \mathrm{ClNO}_{3}[\mathrm{M}+\mathrm{H}]^{+}: 304.0735$, found 304.0741 .

3-羰基-3-(3-三氟甲基苯胺基)丙酸甲酯(2e $)^{[22]}$ : 白 色固体, 产率 89\%. m.p. 63 64 ${ }^{\circ} \mathrm{C} ;{ }^{1} \mathrm{H}$ NMR $(300 \mathrm{MHz}$, $\left.\mathrm{CDCl}_{3}\right) \delta: 9.46(\mathrm{~s}, 1 \mathrm{H}, \mathrm{CONH}), 7.87(\mathrm{~s}, 1 \mathrm{H}, \mathrm{ArH}), 7.75(\mathrm{~d}$, $J=7.9 \mathrm{~Hz}, 1 \mathrm{H}, \mathrm{ArH}), 7.55 \sim 7.30(\mathrm{~m}, 2 \mathrm{H}, \mathrm{ArH}), 3.81(\mathrm{~s}$, $\left.3 \mathrm{H}, \mathrm{OCH}_{3}\right), 3.52\left(\mathrm{~s}, 2 \mathrm{H}, \mathrm{COCH}_{2} \mathrm{CO}\right)$.

3-羰基-3-(2-氰基苯胺基)丙酸甲酯 $(2 \mathrm{f})^{[23]}$ : 淡黄色 固体, 产率 86\%. m.p. 98 99 ${ }^{\circ} \mathrm{C} ;{ }^{1} \mathrm{H}$ NMR (300 MHz, $\left.\mathrm{CDCl}_{3}\right) \delta: 9.79$ (s, $\left.1 \mathrm{H}, \mathrm{CONH}\right), 8.32(\mathrm{~d}, J=8.3 \mathrm{~Hz}, 1 \mathrm{H}$, ArH), $7.78 \sim 7.38(\mathrm{~m}, 2 \mathrm{H}, \mathrm{ArH}), 7.22 \sim 7.02(\mathrm{~m}, 1 \mathrm{H}, \mathrm{ArH})$, $3.81\left(\mathrm{~s}, 3 \mathrm{H}, \mathrm{OCH}_{3}\right), 3.57$ (s, $\left.2 \mathrm{H}, \mathrm{COCH}_{2} \mathrm{CO}\right)$. 
3-羰基-3-(4-乙氧基苯胺基)丙酸甲酯(2g): 白色固 体, 产率 84\%. m.p. 109 $110{ }^{\circ} \mathrm{C} ;{ }^{1} \mathrm{H}$ NMR (300 MHz, $\left.\mathrm{CDCl}_{3}\right) \delta: 9.03(\mathrm{~s}, 1 \mathrm{H}, \mathrm{CONH}), 7.61 \sim 7.37(\mathrm{~m}, 2 \mathrm{H}, \mathrm{ArH})$, $7.94 \sim 7.64(\mathrm{~m}, 2 \mathrm{H}, \operatorname{ArH}), 4.01(\mathrm{q}, J=7.0 \mathrm{~Hz}, 2 \mathrm{H}$, $\left.\mathrm{ArOCH}_{2}\right), 3.79\left(\mathrm{~s}, 3 \mathrm{H}, \mathrm{OCH}_{3}\right), 3.47$ (s, 2H, $\left.\mathrm{COCH}_{2} \mathrm{CO}\right)$, $1.40\left(\mathrm{t}, J=7.0 \mathrm{~Hz}, 3 \mathrm{H}, \mathrm{CH}_{2} \mathrm{CH}_{3}\right) ;{ }^{13} \mathrm{C} \mathrm{NMR}(75 \mathrm{MHz}$, $\left.\mathrm{CDCl}_{3}\right) \delta: 169.98,162.58,155.80,130.3$ 0, 121.73, 114.56, 63.49, 52.36, 41.28, 14.61. HRMS (ESI) calcd for $\mathrm{C}_{12} \mathrm{H}_{16} \mathrm{NO}_{4}[\mathrm{M}+\mathrm{H}]^{+}:$238.1074, found 238. 1073.

3-羰基-3-(4-甲氧基苯胺基)丙酸甲酯(2h): 淡棕色 液体, 产率 $86 \% .{ }^{1} \mathrm{H} \mathrm{NMR}\left(300 \mathrm{MHz}, \mathrm{CDCl}_{3}\right) \delta: 9.25(\mathrm{~s}$, 1H, CONH), 7.22 (t, $J=2.2 \mathrm{~Hz}, 1 \mathrm{H}, \mathrm{ArH}), 7.11$ (t, $J=8.1$ $\mathrm{Hz}, 1 \mathrm{H}, \mathrm{ArH}), 7.02 \sim 6.94(\mathrm{~m}, 1 \mathrm{H}, \mathrm{ArH}), 6.77 \sim 6.36(\mathrm{~m}$, $1 \mathrm{H}, \mathrm{ArH}), 3.66\left(\mathrm{~s}, 3 \mathrm{H}, \mathrm{OCH}_{3}\right), 3.65\left(\mathrm{~s}, 3 \mathrm{H}, \mathrm{OCH}_{3}\right), 3.41$ $\left(\mathrm{s}, 2 \mathrm{H}, \mathrm{COCH}_{2} \mathrm{CO}\right) ;{ }^{13} \mathrm{C} \mathrm{NMR}\left(75 \mathrm{MHz}, \mathrm{CDCl}_{3}\right) \delta: 169.11$, 163.76, 159.86, 138.6 3, 129.41, 112.2 , 110.19, 105.8, 54.98, 52.28, 42.25; HRMS (ESI) calcd for $\mathrm{C}_{11} \mathrm{H}_{14} \mathrm{NO}_{4}$ $[\mathrm{M}+\mathrm{H}]^{+}: 224.0917$, found 224.0921.

3-羰基-3-(2-甲氧基苯胺基)丙酸甲酯(2i $)^{[24]}$ : 无色晶 体, 产率 87\%. m.p. $76 \sim 77{ }^{\circ} \mathrm{C}$; ${ }^{1} \mathrm{H}$ NMR $(300 \mathrm{MHz}$, $\mathrm{CDCl} 3) \delta: 9.33(\mathrm{~s}, 1 \mathrm{H}, \mathrm{CONH}), 8.32(\mathrm{dd}, J=8.0,1.6 \mathrm{~Hz}$, $1 \mathrm{H}, \mathrm{ArH}), 7.08 \sim 6.96(\mathrm{~m}, 1 \mathrm{H}, \mathrm{ArH}), 6.96 \sim 6.77(\mathrm{~m}, 2 \mathrm{H}$, $\mathrm{ArH}), 3.85$ (s, 3H, $\left.\mathrm{OCH}_{3}\right), 3.74\left(\mathrm{~s}, 3 \mathrm{H}, \mathrm{OCH}_{3}\right), 3.47$ (s, 2H, $\left.\mathrm{COCH}_{2} \mathrm{CO}\right)$.

3-羰基-3-(4-甲基苯胺基)丙酸甲酯 $(2 \mathbf{j})^{[25]}$ : 无色晶 体, 产率 92\%. m.p. 88 89 ${ }^{\circ} \mathrm{C} ;{ }^{1} \mathrm{H}$ NMR $(300 \mathrm{MHz}$, $\left.\mathrm{CDCl}_{3}\right) \delta: 9.13$ (s, 1H, CONH), 7.49 7.41 (m, 2H, ArH), $7.19 \sim 7.10(\mathrm{~m}, 2 \mathrm{H}, \mathrm{ArH}), 3.81\left(\mathrm{~s}, 3 \mathrm{H}, \mathrm{OCH}_{3}\right), 3.49(\mathrm{~s}, 2 \mathrm{H}$, $\mathrm{COCH}_{2} \mathrm{CO}$ ), 2.34 (s, $3 \mathrm{H}, \mathrm{ArCH}_{3}$.

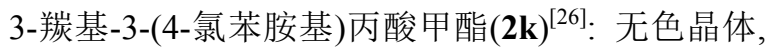
产率 89\%. m.p. $115 \sim 116{ }^{\circ} \mathrm{C}$ (文献值 $106 \sim 108{ }^{\circ} \mathrm{C}$ ); ${ }^{1} \mathrm{H}$ NMR (300 MHz, $\left.\mathrm{CDCl}_{3}\right) \delta: 9.30(\mathrm{~s}, 1 \mathrm{H}, \mathrm{CONH}), 7.52 \sim$ $7.43(\mathrm{~m}, 2 \mathrm{H}, \mathrm{ArH}), 7.29 \sim 7.22$ (m, 2H, ArH), 3.77 (s, 3H, $\left.\mathrm{OCH}_{3}\right), 3.48$ (s, 2H, $\left.\mathrm{COCH}_{2} \mathrm{CO}\right)$.

3-羰基-3-(5-氟-2-甲基苯胺基)丙酸甲酯(2l): 白色 固体, 产率 82\%. m.p. 89 90 ${ }^{\circ} \mathrm{C} ;{ }^{1} \mathrm{H}$ NMR $(300 \mathrm{MHz}$, $\left.\mathrm{CDCl}_{3}\right) \delta: 9.32(\mathrm{~s}, 1 \mathrm{H}, \mathrm{CONH}), 7.91(\mathrm{dd}, J=11.0,2.7 \mathrm{~Hz}$, $1 \mathrm{H}, \mathrm{ArH}), 7.16 \sim 6.98(\mathrm{~m}, 1 \mathrm{H}, \mathrm{ArH}), 6.86 \sim 6.65(\mathrm{~m}, 1 \mathrm{H}$, $\mathrm{ArH}), 3.82\left(\mathrm{~s}, 3 \mathrm{H}, \mathrm{OCH}_{3}\right), 3.51\left(\mathrm{~s}, 2 \mathrm{H}, \mathrm{COCH}_{2} \mathrm{CO}\right), 2.28$ $\left(\mathrm{s}, 3 \mathrm{H}, \mathrm{ArCH}_{3}\right) ;{ }^{13} \mathrm{C} \mathrm{NMR}\left(75 \mathrm{MHz}, \mathrm{CDCl}_{3}\right) \delta: 170.49$, $162.65,159.46(\mathrm{~d}, J=239.6 \mathrm{~Hz}), 136.56(\mathrm{~d}, J=11.2 \mathrm{~Hz})$, 130.78 (d, $J=9.0 \mathrm{~Hz}), 122.81(\mathrm{~d}, J=3.0 \mathrm{~Hz}), 110.84$ (d, $J=21.3 \mathrm{~Hz}$ ), 108.60 (d, $J=26.8 \mathrm{~Hz}$ ), 52.49, 40.74, 16.83; HRMS (ESI) calcd for $\mathrm{C}_{11} \mathrm{H}_{13} \mathrm{FNO}_{3}[\mathrm{M}+\mathrm{H}]^{+}:$226.0874, found 226.0876 . 3-羰基-3-(3-氯-4-甲基苯胺基)丙酸甲酯(2m) ${ }^{[27]}$ : 黄 色固体，产率 88\%. m.p. 93 94 ${ }^{\circ} \mathrm{C} ;{ }^{1} \mathrm{H}$ NMR (300 MHz, DMSO- $\left.d_{6}\right) \delta: 10.27(\mathrm{~s}, 1 \mathrm{H}, \mathrm{CONH}), 7.76(\mathrm{~d}, J=2.0 \mathrm{~Hz}$, $1 \mathrm{H}, \mathrm{ArH}), 7.37 \sim 7.22(\mathrm{~m}, 2 \mathrm{H}, \mathrm{ArH}), 3.64\left(\mathrm{~s}, 3 \mathrm{H}, \mathrm{OCH}_{3}\right)$, 3.46 (s, $\left.2 \mathrm{H}, \mathrm{COCH}_{2} \mathrm{CO}\right), 2.25$ (s, $\left.3 \mathrm{H}, \mathrm{ArCH}_{3}\right)$.

3-羰基-3-(4-溴-3-三氟甲基苯胺基)丙酸甲酯(2n): 白色固体, 产率 83\%. m.p. 78 $79{ }^{\circ} \mathrm{C} ;{ }^{1} \mathrm{H}$ NMR (300 $\left.\mathrm{MHz}, \mathrm{CDCl}_{3}\right) \delta: 9.51$ (s, 1H, CONH), 7.87 (d, $J=2.3 \mathrm{~Hz}$, $1 \mathrm{H}, \mathrm{ArH}), 7.76 \sim 7.54(\mathrm{~m}, J=15.8,5.5 \mathrm{~Hz}, 2 \mathrm{H}, \mathrm{ArH}), 3.82$ $\left(\mathrm{s}, 3 \mathrm{H}, \mathrm{OCH}_{3}\right), 3.50\left(\mathrm{~s}, 2 \mathrm{H}, \mathrm{COCH}_{2} \mathrm{CO}\right) ;{ }^{13} \mathrm{C} \mathrm{NMR}(75$ $\left.\mathrm{MHz}, \mathrm{DMSO}-d_{6}\right) \delta$ : 167.98, 164.99, 138.91, 135.83, 128.91 (q, $J=30.7 \mathrm{~Hz}), 124.19,123.02$ (q, $J=271.6 \mathrm{~Hz})$, 118.31 (q, $J=6.0 \mathrm{~Hz}$ ), 112.07, 52.29, 43.77; HRMS (ESI) calcd for $\mathrm{C}_{12} \mathrm{H}_{16} \mathrm{NO}_{4}[\mathrm{M}+\mathrm{H}]^{+}:$339.9791, found 339.9792.

\subsection{2 中间体 3 的合成}

中间体 3a 的制备参考文献[28]. 取中间体 2 (10 $\mathrm{mmol}$ ), 四氢呋喃 $20 \mathrm{~mL}$ 溶解, 后加入一水合氢氧化锂 $(0.84 \mathrm{~g}, 20 \mathrm{mmol})$ 水溶液 $20 \mathrm{~mL}$, 反应约 $1 \mathrm{~h}$ 后, TLC 检测 反应完全, 加稀盐酸中和至酸性, 乙酸乙酯和水萃取, 有机相干燥后浓缩, 乙酸乙酯和石油醚重结晶得中间体 3.

3-羰基-3-(2,5-二三氟甲基苯胺基)丙酸(3a)：白色固 体, 产率 87\%. m.p. $154 \sim 155{ }^{\circ} \mathrm{C}$; ${ }^{1} \mathrm{H}$ NMR (300 MHz, DMSO- $\left.d_{6}\right) \delta$ : $10.18(\mathrm{~s}, 1 \mathrm{H}, \mathrm{CONH}), 8.08$ (s, 1H, ArH), $7.98(\mathrm{~d}, J=8.2 \mathrm{~Hz}, 1 \mathrm{H}, \operatorname{ArH}), 7.78$ (d, $J=8.2 \mathrm{~Hz}, 1 \mathrm{H}$, $\mathrm{ArH}), 3.50$ (s, 2H, $\left.\mathrm{COCH}_{2} \mathrm{CO}\right) ;{ }^{13} \mathrm{C} \mathrm{NMR}(75 \mathrm{MHz}$, DMSO- $\left.d_{6}\right) \delta: 169.59,166.07,136.30,133.17$ (q, $J=32.5$ $\mathrm{Hz}), 128.01$ (q, $J=5.2 \mathrm{~Hz}), 126.42$ (q, $J=30.3 \mathrm{~Hz})$, $124.68,122.77$ (q, $J=3.4 \mathrm{~Hz}), 121.41$ (q, $J=271.2 \mathrm{~Hz}$ ), 121.05 (q, $J=272.2 \mathrm{~Hz}$ ), 42.63; HRMS (ESI) calcd for $\mathrm{C}_{11} \mathrm{H}_{8} \mathrm{~F}_{6} \mathrm{NO}_{3}[\mathrm{M}+\mathrm{H}]^{+}: 316.0403$, found 316.0406.

3-羰基-3-(2-氯-4-三氟甲基苯胺基)丙酸(3b): 白色 固体, 产率 86\%. m.p. 133 $134{ }^{\circ} \mathrm{C} ;{ }^{1} \mathrm{H}$ NMR $(300 \mathrm{MHz}$, DMSO- $\left.d_{6}\right) \delta: 12.84$ (s, 1H, COOH), 10.09 (s, 1H, CONH), $8.21(\mathrm{~d}, J=8.6 \mathrm{~Hz}, 1 \mathrm{H}, \operatorname{ArH}), 7.91(\mathrm{~d}, J=1.4 \mathrm{~Hz}, 1 \mathrm{H}$, ArH), 7.71 (dd, $J=8.7,1.6 \mathrm{~Hz}, 1 \mathrm{H}, \mathrm{ArH}), 3.55$ (s, 2H, $\left.\mathrm{COCH}_{2} \mathrm{CO}\right) ;{ }^{13} \mathrm{C}$ NMR $\left(75 \mathrm{MHz}, \mathrm{DMSO}-d_{6}\right) \delta: 169.89$, $165.72,138.74,126.68(\mathrm{q}, J=3.9 \mathrm{~Hz}), 125.82$ (q, $J=32.7 \mathrm{~Hz}), 124.82$ (q, $J=3.5 \mathrm{~Hz}), 123.61$ (q, $J=270.3$ $\mathrm{Hz}$ ), 124.16, 43.23; HRMS (ESI) calcd for $\mathrm{C}_{10} \mathrm{H}_{8} \mathrm{ClF}_{3} \mathrm{NO}_{3}$ $[\mathrm{M}+\mathrm{H}]^{+}:$282.0139, found 282.0142.

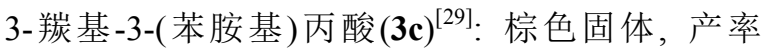
$95 \%$. m.p. $122 \sim 123{ }^{\circ} \mathrm{C}$ (文献值 $\left.163 \sim 165{ }^{\circ} \mathrm{C}\right) ;{ }^{1} \mathrm{H}$ NMR $\left(300 \mathrm{MHz}, \mathrm{DMSO}-d_{6}\right) \delta: 10.15(\mathrm{~s}, 1 \mathrm{H}, \mathrm{CONH}), 7.73 \sim$ 7.42 (m, 2H, ArH), $7.42 \sim 7.16(\mathrm{~m}, 2 \mathrm{H}, \mathrm{ArH}), 7.16 \sim 6.90$ 
(m, $1 \mathrm{H}, \mathrm{ArH}), 3.34$ (s, 2H, $\left.\mathrm{COCH}_{2} \mathrm{CO}\right)$.

3-羰基-3-(4-氯联苯-2-胺基)丙酸(3d)：白色固体, 产率 82\%. m.p. $138 \sim 139{ }^{\circ} \mathrm{C} ;{ }^{1} \mathrm{H}$ NMR $(300 \mathrm{MHz}$, DMSO- $\left.d_{6}\right) \delta: 9.55(\mathrm{~s}, 1 \mathrm{H}, \mathrm{CONH}), 7.63(\mathrm{~d}, J=7.9 \mathrm{~Hz}, 1 \mathrm{H}$, $\mathrm{ArH}$ ), $7.55 \sim 7.20$ (m, 7H, $\mathrm{ArH}), 3.29$ (s, $2 \mathrm{H}, \mathrm{COCH}_{2} \mathrm{CO}$ ); ${ }^{13} \mathrm{C}$ NMR (75 MHz, DMSO- $d_{6}$ ) $\delta: 169.92,165.47,137.80$, $134.99,134.83,132.63,131.27,130.61,128.85,128.53$, 126.60, 126.32, 43.34; HRMS (ESI) calcd for $\mathrm{C}_{15} \mathrm{H}_{12} \mathrm{Cl}-$ $\mathrm{NO}_{3}[\mathrm{M}+\mathrm{H}]^{+}: 290.0578$, found 290.0375 .

3-羰基-3-(3-三氟甲基苯胺基)丙酸 (3e $)^{[22]}$ : 白色固 体, 产率 86\%. m.p. 104 105 ${ }^{\circ} \mathrm{C} ;{ }^{1} \mathrm{H}$ NMR $(300 \mathrm{MHz}$, DMSO- $\left.d_{6}\right) \delta: 12.72(\mathrm{~s}, 1 \mathrm{H}, \mathrm{COOH}), 10.47(\mathrm{~s}, 1 \mathrm{H}, \mathrm{CONH})$, 8.09 (s, 1H, ArH), 7.75 (d, $J=8.5 \mathrm{~Hz}, 1 \mathrm{H}, \operatorname{ArH}), 7.53$ (d, $J=8.0 \mathrm{~Hz}, 1 \mathrm{H}, \mathrm{ArH}), 7.45 \sim 7.21(\mathrm{~m}, 1 \mathrm{H}, \mathrm{ArH}), 3.41(\mathrm{~s}$, $\left.2 \mathrm{H}, \mathrm{COCH}_{2} \mathrm{CO}\right)$.

3-羰基-3-(2-氰基苯胺基)丙酸 $(3 \mathrm{f})^{[30]}$ : 浅黄色固体, 产率 $89 \%$. m.p. $136 \sim 137{ }^{\circ} \mathrm{C}$ (文献值 $114 \sim 116{ }^{\circ} \mathrm{C}$ ); ${ }^{1} \mathrm{H}$ NMR (300 MHz, DMSO- $\left.d_{6}\right) \delta: 12.68(\mathrm{~s}, 1 \mathrm{H}, \mathrm{COOH})$, 10.37 (s, $1 \mathrm{H}, \mathrm{CONH}), 7.81 \sim 7.65(\mathrm{~m}, 3 \mathrm{H}, \mathrm{ArH}), 7.36 \sim$ 7.30 (m, $1 \mathrm{H}, \mathrm{ArH}), 3.48$ (s, $\left.2 \mathrm{H}, \mathrm{COCH}_{2} \mathrm{CO}\right)$.

3-羰基-3-(4-乙氧基苯胺基)丙酸(3g $)^{[31]}$ : 白色固体, 产率 90\%. m.p. $144 \sim 145{ }^{\circ} \mathrm{C}$; ${ }^{1} \mathrm{H}$ NMR $(300 \mathrm{MHz}$, DMSO- $\left.d_{6}\right) \delta: 10.03(\mathrm{~s}, 1 \mathrm{H}, \mathrm{COOH}), 8.16 \sim 7.29(\mathrm{~m}, 2 \mathrm{H}$, ArH), $7.04 \sim 6.76(\mathrm{~m}, 2 \mathrm{H}, \mathrm{ArH}), 4.01(\mathrm{q}, J=6.9 \mathrm{~Hz}, 2 \mathrm{H}$, $\left.\mathrm{ArOCH}_{2}\right), 3.35$ (s, $\left.2 \mathrm{H}, \mathrm{COCH}_{2} \mathrm{CO}\right), 1.34(\mathrm{t}, J=7.0 \mathrm{~Hz}, 3 \mathrm{H}$, $\mathrm{CH}_{2} \mathrm{CH}_{3}$ ).

3-羰基-3-(4-甲氧基苯胺基)丙酸(3h) ${ }^{[29]}$ : 无色晶体, 产率 84\%. m.p. $94 \sim 95{ }^{\circ} \mathrm{C} ;{ }^{1} \mathrm{H}$ NMR $(300 \mathrm{MHz}$, DMSO- $\left.d_{6}\right) \delta: 12.72(\mathrm{~s}, 1 \mathrm{H}, \mathrm{COOH}), 10.19(\mathrm{~s}, 1 \mathrm{H}, \mathrm{CONH})$, $7.36(\mathrm{t}, J=2.2 \mathrm{~Hz}, 1 \mathrm{H}, \mathrm{ArH}), 7.28(\mathrm{t}, J=8.1 \mathrm{~Hz}, 1 \mathrm{H}, \mathrm{ArH})$, $7.21 \sim 7.14(\mathrm{~m}, 1 \mathrm{H}, \mathrm{ArH}), 6.75 \sim 6.67(\mathrm{~m}, 1 \mathrm{H}, \mathrm{ArH}), 3.79$ (s, $\left.3 \mathrm{H}, \mathrm{OCH}_{3}\right), 3.42\left(\mathrm{~s}, 2 \mathrm{H}, \mathrm{COCH}_{2} \mathrm{CO}\right)$.

3-羰基-3-(2-甲氧基苯胺基)丙酸 (3i) ${ }^{[29]}$ : 白色固体, 产率 86\%. m.p. $147 \sim 148{ }^{\circ} \mathrm{C}$; ${ }^{1} \mathrm{H}$ NMR $(300 \mathrm{MHz}$, DMSO- $\left.d_{6}\right) \delta: 12.71(\mathrm{~s}, 1 \mathrm{H}, \mathrm{COOH}), 9.57(\mathrm{~s}, 1 \mathrm{H}, \mathrm{CONH})$, 8.11 (dd, $J=8.0,1.3 \mathrm{~Hz}, 1 \mathrm{H}, \operatorname{ArH}), 7.15 \sim 7.01(\mathrm{~m}, 2 \mathrm{H}$, $\mathrm{ArH}), 6.98 \sim 6.87(\mathrm{~m}, 1 \mathrm{H}, \mathrm{ArH}), 3.84\left(\mathrm{~s}, 3 \mathrm{H}, \mathrm{ArOCH}_{3}\right)$, $3.54\left(\mathrm{~s}, 2 \mathrm{H}, \mathrm{COCH}_{2} \mathrm{CO}\right)$.

3-羰基-3-(4-甲基苯胺基)丙酸 $(\mathbf{3 j})^{[22]}$ : 白色固体, 产 率 91\%. m.p. $119 \sim 120{ }^{\circ} \mathrm{C}$; ${ }^{1} \mathrm{H}$ NMR $(300 \mathrm{MHz}$, DMSO- $\left.d_{6}\right) \delta: 12.59$ (s, $\left.1 \mathrm{H}, \mathrm{COOH}\right), 10.03(\mathrm{~s}, 1 \mathrm{H}, \mathrm{CONH})$, 7.49 (d, $J=8.4 \mathrm{~Hz}, 2 \mathrm{H}, \mathrm{ArH}), 7.12(\mathrm{~d}, J=8.2 \mathrm{~Hz}, 2 \mathrm{H}$, $\mathrm{ArH}), 3.36$ (s, $\left.2 \mathrm{H}, \mathrm{COC} \mathrm{H}_{2} \mathrm{CO}\right), 2.26$ (s, 3H, $\mathrm{ArCH}_{3}$ ).

3 -羰基-3-(4-氯苯胺基)丙酸 $(3 \mathbf{k})^{[26]}$ : 白色固体, 产 率 87\%. m.p. $145 \sim 146{ }^{\circ} \mathrm{C} ;{ }^{1} \mathrm{H}$ NMR $(300 \mathrm{MHz}$, DMSO- $\left.d_{6}\right) \delta: 12.64(\mathrm{~s}, 1 \mathrm{H}, \mathrm{COOH}), 10.24(\mathrm{~s}, 1 \mathrm{H}, \mathrm{CONH}), 7.70 \sim$ 7.54 (m, 2H, ArH), 7. 48 7.23 (m, 2H, ArH), $3.36(\mathrm{~s}, 2 \mathrm{H}$, $\left.\mathrm{COCH}_{2} \mathrm{CO}\right)$.

3-羰基-3-(5-氟-2-甲基苯胺基)丙酸(3I): 白色固体, 产率 82\%. m.p. $132 \sim 133{ }^{\circ} \mathrm{C} ;{ }^{1} \mathrm{H}$ NMR $(300 \mathrm{MHz}$, DMSO- $\left.d_{6}\right) \delta: 12.73(\mathrm{~s}, 1 \mathrm{H}, \mathrm{COOH}), 9.58(\mathrm{~s}, 1 \mathrm{H}, \mathrm{CONH})$, 7.49 (dd, $J=11.1 \mathrm{~Hz}, 2.7 \mathrm{~Hz}, 1 \mathrm{H}, \mathrm{ArH}), 7.35 \sim 7.08(\mathrm{~m}$, $1 \mathrm{H}, \mathrm{ArH}), 7.11 \sim 6.68(\mathrm{~m}, 1 \mathrm{H}, \mathrm{ArH}), 3.47(\mathrm{~s}, 2 \mathrm{H}$, $\mathrm{COCH}_{2} \mathrm{CO}$ ), 2.22 (s, 3H, $\left.\mathrm{ArCH}_{3}\right) ;{ }^{13} \mathrm{C}$ NMR $(75 \mathrm{MHz}$, DMSO- $\left.d_{6}\right) \delta: 169.80,165.17,161.97(\mathrm{~d}, J=238.3 \mathrm{~Hz})$, 137.63 (d, $J=10.8 \mathrm{~Hz}), 131.65$ (d, $J=9.0 \mathrm{~Hz}), 126.25$ (d, $J=2.9 \mathrm{~Hz}), 111.46(\mathrm{~d}, J=20.8 \mathrm{~Hz}), 110.50$ (d, $J=24.9$ $\mathrm{Hz}$ ), 43.47, 17.27; HRMS (ESI) calcd for $\mathrm{C}_{10} \mathrm{H}_{11} \mathrm{FNO}_{3}$ $[\mathrm{M}+\mathrm{H}]^{+}:$212.0717, found 212.0718.

3-羰基-3-(3-氯-4-甲基苯胺基)丙酸 $(3 \mathrm{~m})^{[27]}$ : 黄色固 体, 产率 $89 \%$. m.p. 142 143 ${ }^{\circ} \mathrm{C} ;{ }^{1} \mathrm{H}$ NMR $(300 \mathrm{MHz}$, DMSO- $\left.d_{6}\right) \delta: 12.66$ (s, $\left.1 \mathrm{H}, \mathrm{COOH}\right), 10,21(\mathrm{~s}, 1 \mathrm{H}, \mathrm{CONH})$, $7.78(\mathrm{~d}, J=1.6 \mathrm{~Hz}, 1 \mathrm{H}, \mathrm{ArH}), 7.38 \sim 7.17(\mathrm{~m}, 2 \mathrm{H}, \mathrm{ArH})$, 3.34 (s, $\left.2 \mathrm{H}, \mathrm{COCH}_{2} \mathrm{CO}\right), 2.24$ (s, $3 \mathrm{H}, \mathrm{ArCH}_{3}$ ).

3-羰基-3-(4-溴-3-三氟甲基苯胺基)丙酸(3n): 白色 固体, 产率 83\%. m.p. $149 \sim 150{ }^{\circ} \mathrm{C} ;{ }^{1} \mathrm{H}$ NMR $(300 \mathrm{MHz}$, DMSO- $\left.d_{6}\right) \delta: 12.82(\mathrm{~s}, 1 \mathrm{H}, \mathrm{COOH}), 10.67(\mathrm{~s}, 1 \mathrm{H}, \mathrm{CONH})$, 8.24 (d, $J=2.2 \mathrm{~Hz}, 1 \mathrm{H}, \mathrm{ArH}), 7.93 \sim 7.73(\mathrm{~m}, 2 \mathrm{H}, \mathrm{ArH})$, 3.49 (s, $2 \mathrm{H}, \mathrm{COCH}_{2} \mathrm{CO}$ ); ${ }^{13} \mathrm{C}$ NMR (75 MHz, DMSO- $d_{6}$ ) $\delta: 169.13,165.52,139.04,135.75,128.90$ (q, $J=30.7 \mathrm{~Hz})$, $124.09,123.02$ (q, $J=271.5 \mathrm{~Hz}), 118.25(\mathrm{q}, J=5.8 \mathrm{~Hz})$, 111.89, 44.31; HRMS (ESI) calcd for $\mathrm{C}_{10} \mathrm{H}_{8} \mathrm{BrF}_{3} \mathrm{NO}_{3}[\mathrm{M}+$ $\mathrm{H}]^{+}:$325.9634, found 325.9632 .

\subsection{3 $\mathrm{N}$-(2,6-二甲基苯基)丙酸甲酯(6)的合成}

中间体 6 的制备参考文献[33]. 在 $250 \mathrm{~mL}$ 圆底烧瓶 中加入 2,6-二甲基苯胺 $(1.21 \mathrm{~g}, 10 \mathrm{mmol}$ )、碳酸氢钠 $(1.00$ $\mathrm{g}, 12 \mathrm{mmol}) 、 2$-溴丙酸甲酯 $(5.01 \mathrm{~g}, 30 \mathrm{mmol}), 122{ }^{\circ} \mathrm{C}$ 反 应过夜, TLC 检测反应完全, 过滤, 浓缩后柱层析分析 分离得 $1.96 \mathrm{~g}$ 棕色液体 $\mathbf{6}^{[32]}$, 产率 95\%. ${ }^{1} \mathrm{H}$ NMR (300 MHz, DMSO- $\left.d_{6}\right) \delta: 7.02(\mathrm{~d}, J=7.4 \mathrm{~Hz}, 1 \mathrm{H}, \mathrm{ArH}), 6.85(\mathrm{t}$, $J=7.4 \mathrm{~Hz}, 2 \mathrm{H}, \mathrm{ArH}), 4.04$ (q, $J=7.0 \mathrm{~Hz}, 1 \mathrm{H}, \mathrm{COCH}$ ), $3.83(\mathrm{~s}, 1 \mathrm{H}, \mathrm{NH}), 3.72\left(\mathrm{~s}, 3 \mathrm{H}, \mathrm{OCH}_{3}\right), 2.36(\mathrm{~s}, 6 \mathrm{H}, 2 \times$ ArMe), 1.44 (d, $J=6.9 \mathrm{~Hz}, 3 \mathrm{H}, \mathrm{CHCH}_{3}$ ).

3.3.4 目标化合物 7 的合成

目标化合物 7 的制备参考文献[17]. $250 \mathrm{~mL}$ 圆底烧 瓶中, 加入中间体 $3(10 \mathrm{mmol})$, 干燥二氯甲烷 $20 \mathrm{~mL}, 3$ 滴 DMF，在冰浴和氮气保护条件下，滴加草酰氯 $(1.27 \mathrm{~g}$, $10 \mathrm{mmol}$ ), 滴加完毕后, 室温反应 $2 \sim 3 \mathrm{~h}$, 得中间体 $\mathbf{4}$; 在冰浴和氮气保护条件下, 加入碳酸氢钠 $(1.26 \mathrm{~g}, 15$ $\mathrm{mmol})$, 将中间体 4 滴加至化合物 $6(2.07 \mathrm{~g}, 10 \mathrm{mmol})$ 的 
甲苯溶液中, $4 \mathrm{~h}$ 后, TLC 检测反应完全, 甲醇终止反应, 浓缩除去甲苯, 二氯甲烷和水萃取, 有机相干燥, 浓缩 后柱层析得目标化合物 7 .

2-[ $N$-(2,6-二甲基苯基)-3-羰基-3-(2,5-二三氟甲基苯 胺基)丙酰胺基]丙酸甲酯(7a): 淡棕色固体, 产率 75\%. m.p. 89 90 ${ }^{\circ} \mathrm{C} ;{ }^{1} \mathrm{H}$ NMR $\left(300 \mathrm{MHz}, \mathrm{CDCl}_{3}\right) \delta: 10.71$ (s, $1 \mathrm{H}, \mathrm{CONH}), 8.56(\mathrm{~s}, 1 \mathrm{H}, \mathrm{ArH}), 7.75(\mathrm{~d}, J=8.3 \mathrm{~Hz}, 1 \mathrm{H}$, ArH), $7.45(\mathrm{~d}, J=8.2 \mathrm{~Hz}, 1 \mathrm{H}, \mathrm{ArH}), 7.37 \sim 7.06(\mathrm{~m}, 3 \mathrm{H}$, $\mathrm{ArH}), 4.55$ (q, $J=7.5 \mathrm{~Hz}, 1 \mathrm{H}, \mathrm{CHCO}), 3.81(\mathrm{~s}, 3 \mathrm{H}$, $\mathrm{COOCH}_{3}$ ), 3.11 (dd, $J=18.1,27.2 \mathrm{~Hz}, 2 \mathrm{H}, \mathrm{COCH}_{2} \mathrm{CO}$ ), 2.45, $2.16(2 \mathrm{~s}, 6 \mathrm{H}, 2 \times \mathrm{ArMe}), 1.07(\mathrm{~d}, J=7.4 \mathrm{~Hz}, 3 \mathrm{H}$, $\left.\mathrm{CHCH}_{3}\right) ;{ }^{13} \mathrm{C}$ NMR (75 MHz, CDCl3) $\delta: 171.81,169.05$, $164.16,137.75,136.58,135.81,135.70,134.11$ (q, $J=33.0$ $\mathrm{Hz}), 129.18,128.98,128.55,126.39$ (q, $J=5.3 \mathrm{~Hz}), 122.91$ (q, $J=30.8 \mathrm{~Hz}), 120.97$ (q, $J=271.2 \mathrm{~Hz}), 120.88$ (q, $J=$ $271.7 \mathrm{~Hz}), 120.52$ (q, $J=18.3 \mathrm{~Hz}), 120.32$ (q, $J=3.2 \mathrm{~Hz}$ ), 55.49, 51.60, 39.72, 17.96, 17.51, 14.35; HRMS (ESI) calcd for $\mathrm{C}_{23} \mathrm{H}_{23} \mathrm{~F}_{6} \mathrm{~N}_{2}(\mathrm{M}+\mathrm{H})^{+}: 505.1557$, found 505.1556 .

2-[ $N$-(2,6-二甲基苯基)-3-羰基-3-(2-氯-4-三氟甲基 苯胺基)丙酰胺基]丙酸甲酯(7b): 白色固体, 产率 $83 \%$. m.p. $121 \sim 122{ }^{\circ} \mathrm{C} ;{ }^{1} \mathrm{H}$ NMR $\left(300 \mathrm{MHz}, \mathrm{CDCl}_{3}\right) \delta: 10.80$ (s, $1 \mathrm{H}, \mathrm{CONH}), 8.51$ (d, $J=8.6 \mathrm{~Hz}, 1 \mathrm{H}, \mathrm{ArH}), 7.63$ (d, $J=1.8 \mathrm{~Hz}, 1 \mathrm{H}, \mathrm{ArH}), 7.47$ (dd, $J=1.8 \mathrm{~Hz}, 8.8 \mathrm{~Hz}, 1 \mathrm{H}$, ArH), $7.12 \sim 7.32(\mathrm{~m}, 3 \mathrm{H}, \mathrm{ArH}), 4.57$ (q, $J=7.4 \mathrm{~Hz}, 1 \mathrm{H}$, CHCO), 3.81 (s, 3H, $\mathrm{COOCH}_{3}$ ), 3.15 (dd, $J=17.9,25.3$ $\left.\mathrm{Hz}, 2 \mathrm{H}, \mathrm{COCH}_{2} \mathrm{CO}\right), 2.46,2.16(2 \mathrm{~s}, 6 \mathrm{H}, 2 \times \mathrm{ArMe}), 1.08$ (d, $\left.J=7.4 \mathrm{~Hz}, 3 \mathrm{H}, \mathrm{CHCH}_{3}\right) ;{ }^{13} \mathrm{C} \mathrm{NMR}\left(75 \mathrm{MHz}, \mathrm{CDCl}_{3}\right)$ $\delta: 172.05,169.29,164.12,137.98,137.87,136.73,135.92$, $129.45,129.19,128.78,126.02(\mathrm{q}, J=33.3 \mathrm{~Hz}), 126.01$ (q, $J=3.9 \mathrm{~Hz}), 124.29$ (q, $J=3.7 \mathrm{~Hz}), 123.23,123.17$ (q, $J=$ $270.2 \mathrm{~Hz}), 121.16,55.64,52.03,40.34,18.28,17.92$, 14.70; HRMS (ESI) calcd for $\mathrm{C}_{22} \mathrm{H}_{23} \mathrm{ClF}_{3} \mathrm{~N}_{2} \mathrm{O}_{4}(\mathrm{M}+\mathrm{H})^{+}$: 471.1293, found 471.1293.

2-[ $N$-(2,6-二甲基苯基)-3-羊基-3-(苯胺基)丙酰胺基] 丙酸甲酯(7c): 棕色固体, 产率 92\%. m.p. 98 99 ${ }^{\circ} \mathrm{C}$; ${ }^{1} \mathrm{HNMR}\left(300 \mathrm{MHz}, \mathrm{CDCl}_{3}\right) \delta: 9.87$ (s, 1H, CONH), 7.54 (d, $J=7.6 \mathrm{~Hz}, 2 \mathrm{H}, \mathrm{ArH}), 7.25 \sim 7.32(\mathrm{~m}, 2 \mathrm{H}, \mathrm{ArH}), 7.05 \sim$ 7.22 (m, 4H, ArH), 4.51 (q, $J=7.4 \mathrm{~Hz}, 1 \mathrm{H}, \mathrm{CHCO}), 3.82$ $\left(\mathrm{s}, 3 \mathrm{H}, \mathrm{COOCH}_{3}\right), 3.06(\mathrm{dd}, J=17.7,28.8 \mathrm{~Hz}, 2 \mathrm{H}$, $\left.\mathrm{COCH}_{2} \mathrm{CO}\right), 2.14,2.43(2 \mathrm{~s}, 6 \mathrm{H}, 2 \times \mathrm{ArMe}), 1.05$ (d, $J=$ $\left.7.4 \mathrm{~Hz}, 3 \mathrm{H}, \mathrm{CHCH}_{3}\right) ;{ }^{13} \mathrm{C}$ NMR $\left(75 \mathrm{MHz}, \mathrm{CDCl}_{3}\right) \delta$ : $172.52,170.07,163.64,138.16,137.76,136.91,136.24$, $129.65,129.36,129.01,128.84,124.25,120.20,55.85$, 52.40, 40.55, 18.52, 18.24, 15.01; HRMS (ESI) calcd for $\mathrm{C}_{21} \mathrm{H}_{25} \mathrm{~N}_{2} \mathrm{O}_{4}(\mathrm{M}+\mathrm{H})^{+}: 369.1809$, found 369.1812.
2-[ $\mathrm{N}$-(2,6-二甲基苯基)-3-羰基-3-(4-氯联苯-2-胺基) 丙酰胺基]丙酸甲酯(7d): 橘黄色固体, 产率 70\%. m.p. 76 $77{ }^{\circ} \mathrm{C} ;{ }^{1} \mathrm{H}$ NMR $\left(300 \mathrm{MHz}, \mathrm{CDCl}_{3}\right) \delta: 9.77(\mathrm{~s}, 1 \mathrm{H}$, CONH), $8.12(\mathrm{~d}, J=7.9 \mathrm{~Hz}, 1 \mathrm{H}, \mathrm{ArH}), 7.42 \sim 7.46(\mathrm{~m}, 2 \mathrm{H}$, $\mathrm{ArH}), 7.32 \sim 7.38(\mathrm{~m}, 3 \mathrm{H}, \mathrm{ArH}), 7.08 \sim 7.27(\mathrm{~m}, 5 \mathrm{H}, \mathrm{ArH})$, 4.40 (q, $J=7.4 \mathrm{~Hz}, 1 \mathrm{H}, \mathrm{CHCO}), 3.79$ (s, $3 \mathrm{H}, \mathrm{COOCH}_{3}$ ), $2.98\left(\mathrm{dd}, J=17.7,30.7 \mathrm{~Hz}, 2 \mathrm{H}, \mathrm{COCH}_{2} \mathrm{CO}\right), 2.40,2.07$ (2s, 6H, $2 \times$ ArMe), $1.02\left(\mathrm{~d}, J=7.4 \mathrm{~Hz}, 3 \mathrm{H}, \mathrm{CHCH}_{3}\right) ;{ }^{13} \mathrm{C}$ NMR $\left(75 \mathrm{MHz}, \mathrm{CDCl}_{3}\right) \delta: 172.16,169.25,163.84$, $138.08,136.72,136.07,134.57,133.41,132.72,130.67$, $130.04,129.59,129.09,128.72,128.65,128.26,124.70,12$ 2.74, 55.47, 52.05, 40.15, 18.32, 17.99, 14.82; HRMS (ESI) calcd for $\mathrm{C}_{27} \mathrm{H}_{28} \mathrm{ClN}_{2} \mathrm{O}_{4}(\mathrm{M}+\mathrm{H})^{+}: 479.1732$, found 479.1733.

2-[ $N$-(2,6-二甲基苯基)-3-㛶基-3-(3-三氟甲基苯 基)丙酰胺基]丙酸甲酯(7e): 黄绿色液体, 产率 $82 \% .{ }^{1} \mathrm{H}$ NMR (300 MHz, $\left.\mathrm{CDCl}_{3}\right) \delta$ : 9.75 (s, 1H, CONH), 7.42 7.47 (m, 2H, ArH), $7.10 \sim 7.24$ (m, 3H, ArH), 6.79 6.85 $(\mathrm{m}, 2 \mathrm{H}, \mathrm{ArH}), 4.53(\mathrm{q}, J=7.4 \mathrm{~Hz}, 1 \mathrm{H}, \mathrm{CHCO}), 3.82(\mathrm{~s}$, $3 \mathrm{H}, \mathrm{COOCH}_{3}$ ), 3.08 (dd, $J=17.7,28.7 \mathrm{~Hz}, 2 \mathrm{H}$, $\left.\mathrm{COCH}_{2} \mathrm{CO}\right), 2.43,2.14(2 \mathrm{~s}, 6 \mathrm{H}, 2 \times \mathrm{ArMe}), 1.07$ (d, $J=$ $\left.7.4 \mathrm{~Hz}, 3 \mathrm{H}, \mathrm{CHCH}_{3}\right) ;{ }^{13} \mathrm{C}$ NMR $\left(75 \mathrm{MHz}, \mathrm{CDCl}_{3}\right) \delta$ : $172.18,169.60,163.75,138.01,137.79,136.56,135.80,13$ 0.81 (q, $J=32.3 \mathrm{~Hz}), 129.33,129.11,128.99,128.71$, $123.56(\mathrm{q}, J=270.8 \mathrm{~Hz}), 122.75,120.34(\mathrm{q}, J=3.8 \mathrm{~Hz})$, 116.43 (q, $J=4.0 \mathrm{~Hz}$ ), 55.54, 52.07, 40.27, 18.15, 17.88, 14.62; HRMS (ESI) calcd for $\mathrm{C}_{22} \mathrm{H}_{24} \mathrm{~F}_{3} \mathrm{~N}_{2} \mathrm{O}_{4}(\mathrm{M}+\mathrm{H})^{+}$: 437.1683, found 437.1685.

2-[ $\mathrm{N}$-(2,6-二甲基苯基)-3-羰基-3-(2-氧基苯胺基)丙 酰胺基]丙酸甲酯 (7f): 淡棕色固体, 产率 $85 \%$. m.p. 92 93 ${ }^{\circ} \mathrm{C} ;{ }^{1} \mathrm{H}$ NMR $\left(300 \mathrm{MHz}, \mathrm{CDCl}_{3}\right) \delta: 10.72(\mathrm{~s}, 1 \mathrm{H}$, $\mathrm{CONH}), 8.21(\mathrm{~d}, J=8.3 \mathrm{~Hz}, 1 \mathrm{H}, \mathrm{ArH}), 7.61 \sim 7.41(\mathrm{~m}, 2 \mathrm{H}$, ArH), $7.26 \sim 6.96(\mathrm{~m}, 4 \mathrm{H}, \mathrm{ArH}), 4.53(\mathrm{q}, J=7.4 \mathrm{~Hz}, 1 \mathrm{H}$, CHCO), 3.82 (s, $3 \mathrm{H}, \mathrm{COOCH}_{3}$ ), 3.08 (dd, $J=17.7,28.7$ $\left.\mathrm{Hz}, 2 \mathrm{H}, \mathrm{COCH}_{2} \mathrm{CO}\right), 2.43,2.14(2 \mathrm{~s}, 6 \mathrm{H}, 2 \times \mathrm{ArMe}), 1.07$ $\left(\mathrm{d}, J=7.4 \mathrm{~Hz}, 3 \mathrm{H}, \mathrm{CHCH}_{3}\right) ;{ }^{13} \mathrm{C} \mathrm{NMR}\left(75 \mathrm{MHz}, \mathrm{CDCl}_{3}\right) \delta$ : $172.21,169.25,164.27,140.21,138.06,136.80,135.79$, $133.51,132.45,129.44,129.19,128.78,124.15,121.72$, 115.89, 103.47, 55.61, 52.23, 39.96, 18.35, 18.03, 14.75; HRMS (ESI) calcd for $\mathrm{C}_{22} \mathrm{H}_{24} \mathrm{~N}_{3} \mathrm{O}_{4}(\mathrm{M}+\mathrm{H})^{+}: 394.1761$, found 394.1761 .

2-[ $\mathrm{N}$-(2,6-二甲基苯基)-3-羰基-3-(4-乙氧基苯胺基) 丙酰胺基]丙酸甲酯(7g): 黄色液体, 产率 75\%. ${ }^{1} \mathrm{H}$ NMR $\left(300 \mathrm{MHz}, \mathrm{CDCl}_{3}\right) \delta: 9.74$ (s, 1H, CONH), 7.59 7.35 (m, $2 \mathrm{H}, \operatorname{ArH}), 7.30 \sim 7.01(\mathrm{~m}, 3 \mathrm{H}, \mathrm{ArH}), 6.92 \sim 6.73(\mathrm{~m}, 2 \mathrm{H}$, 
$\operatorname{ArH}), 4.52(\mathrm{q}, J=7.5 \mathrm{~Hz}, 1 \mathrm{H}, \mathrm{CHCO}), 4.10 \sim 3.90(\mathrm{~m}, 2 \mathrm{H}$, $\mathrm{ArOCH}_{2}$ ), 3.83 (s, 3H, $\mathrm{COOCH}_{3}$ ), 3.06 (dd, $J=17.9,28.5$ $\left.\mathrm{Hz}, 2 \mathrm{H}, \mathrm{COCH}_{2} \mathrm{CO}\right), 2.43,2.14$ (2s, 6H, $\left.2 \times \mathrm{ArMe}\right), 1.39$ (t, $\left.J=7.0 \mathrm{~Hz}, 3 \mathrm{H}, \mathrm{CH}_{2} \mathrm{CH}_{3}\right), 1.07(\mathrm{~d}, J=7.4 \mathrm{~Hz}, 3 \mathrm{H}$, $\left.\mathrm{CHCH}_{3}\right) ;{ }^{13} \mathrm{C}$ NMR $\left(75 \mathrm{MHz}, \mathrm{CDCl}_{3}\right) \delta: 172.23,169.81$, $163.07,155.36,137.84,136.59,135.94,130.53,129.29$, $128.99,128.65,121.51,114.30,63.27,55.48,52.04$, 40.10, 18.18, 17.91, 14.68, 14.49; HRMS (ESI) calcd for $\mathrm{C}_{23} \mathrm{H}_{29} \mathrm{~N}_{2} \mathrm{O}_{5}(\mathrm{M}+\mathrm{H})^{+}:$413.2071, found 413.2076.

2-[ $N$-(2,6-二甲基苯基)-3-羰基-3-(3-甲氧基苯胺基) 丙酰胺基]丙酸甲酯(7h): 黄色固体, 产率 83\%. m.p. 77 $78{ }^{\circ} \mathrm{C} ;{ }^{1} \mathrm{H}$ NMR $\left(300 \mathrm{MHz}, \mathrm{CDCl}_{3}\right) \delta: 9.85(\mathrm{~s}, 1 \mathrm{H}$, $\mathrm{CONH}$ ), 7.27 (t, $J=2.2 \mathrm{~Hz}, 1 \mathrm{H}, \mathrm{ArH}), 7.24 \sim 7.06(\mathrm{~m}, 5 \mathrm{H}$, $\mathrm{ArH}), 6.61 \sim 6.64(\mathrm{~m}, 1 \mathrm{H}, \mathrm{ArH}), 4.53(\mathrm{q}, J=7.4 \mathrm{~Hz}, 1 \mathrm{H}$, $\mathrm{CHCO}$ ), 3.80 (s, $\left.3 \mathrm{H}, \mathrm{COOCH}_{3}\right), 3.76\left(\mathrm{~s}, 3 \mathrm{H}, \mathrm{ArOCH}_{3}\right)$, 3.09 (dd, $J=17.4,29.3 \mathrm{~Hz}, 2 \mathrm{H}, \mathrm{COCH}_{2} \mathrm{CO}$ ), 2.43, 2.14 (2s, 6H, $2 \times$ ArMe), 1.07 (d, $\left.J=7.5 \mathrm{~Hz}, 3 \mathrm{H}, \mathrm{CHCH}_{3}\right) ;{ }^{13} \mathrm{C}$ NMR $\left(75 \mathrm{MHz}, \mathrm{CDCl}_{3}\right) \delta: 172.2$ 9, 169.78, 163.46, $159.84,138.82,137.96,136.78,136.12,129.41,129.29$, $129.15,128.81,112.18,109.99,105.54,55.66,55.03$, $52.15,40.61,18.32,18.04,14.81$; HRMS (ESI) calcd for $\mathrm{C}_{22} \mathrm{H}_{27} \mathrm{~N}_{2} \mathrm{O}_{5}(\mathrm{M}+\mathrm{H})^{+}: 399.1914$, found 399.1918 .

2-[ $\mathrm{N}$-(2,6-二甲基苯基)-3-羰基-3-(2-甲氧基苯胺基) 丙酰胺基]丙酸甲酯(7i): 棕色液体, 产率 $80 \% .{ }^{1} \mathrm{H}$ NMR $\left(300 \mathrm{MHz}, \mathrm{CDCl}_{3}\right) \delta: 10.00$ (s, 1H, CONH), 8.26 (dd, $J=$ 7.9, $1.6 \mathrm{~Hz}, 1 \mathrm{H}, \mathrm{ArH}), 7.08 \sim 7.27$ (m, 3H, ArH), 6.98 7.04 (m, 1H, ArH), 6.84 6.92 (m, 2H, ArH), 4.53 (q, $J=$ $7.4 \mathrm{~Hz}, 1 \mathrm{H}, \mathrm{CHCO}), 3.87$ (s, $\left.3 \mathrm{H}, \mathrm{OCH}_{3}\right), 3.78(\mathrm{~s}, 3 \mathrm{H}$, $\mathrm{OCH}_{3}$ ), 3.09 (dd, $J=17.1,28.1 \mathrm{~Hz}, 2 \mathrm{H}, \mathrm{COCH}_{2} \mathrm{CO}$ ), 2.42, $2.13(2 \mathrm{~s}, 6 \mathrm{H}, 2 \times \mathrm{ArMe}), 1.05$ (d, $\left.J=7.5 \mathrm{~Hz}, 3 \mathrm{H}, \mathrm{CHCH}_{3}\right)$; ${ }^{13} \mathrm{C}$ NMR $\left(75 \mathrm{MHz}, \mathrm{CDCl}_{3}\right) \delta: 172.28,169.43,163.25$, $148.57,138.08,136.93,136.33,129.34,129.04,128.74$, $127.48,123.76,120.60,120.12,110.07,55.66,55.62$, 51.94, 41.12, 18.37, 18.04, 14.88; HRMS (ESI) calcd for $\mathrm{C}_{22} \mathrm{H}_{27} \mathrm{~N}_{2} \mathrm{O}_{5}(\mathrm{M}+\mathrm{H})^{+}: 399.1914$, found 399.1913 .

2-[ $N$-(2,6-二甲基苯基)-3-羰基-3-(4-甲基苯胺基)丙 酰胺基]丙酸甲酯 $(7 \mathbf{j})$ : 黄色固体, 产率 76\%. m.p. 103 $104{ }^{\circ} \mathrm{C} ;{ }^{1} \mathrm{H}$ NMR $\left(300 \mathrm{MHz}, \mathrm{CDCl}_{3}\right) \delta: 9.82(\mathrm{~s}, 1 \mathrm{H}$, $\mathrm{CONH}$ ), 7.45 (d, $J=8.4 \mathrm{~Hz}, 2 \mathrm{H}, \mathrm{ArH}), 7.10 \sim 7.28(\mathrm{~m}$, $5 \mathrm{H}, \mathrm{ArH}$ ), 4.55 (q, $J=7.4 \mathrm{~Hz}, 1 \mathrm{H}, \mathrm{CHCO}), 3.84$ (s, $3 \mathrm{H}$, $\mathrm{COOCH}_{3}$ ), 3.09 (dd, $J=17.8,29.0 \mathrm{~Hz}, 2 \mathrm{H}, \mathrm{COCH}_{2} \mathrm{CO}$ ), 2.44, 2.31, 2.15 (3s, 9H, $3 \times \mathrm{ArMe}), 1.09$ (d, $J=7.4 \mathrm{~Hz}$, $\left.3 \mathrm{H}, \mathrm{CHCH}_{3}\right) ;{ }^{13} \mathrm{C} \mathrm{NMR}\left(75 \mathrm{MHz}, \mathrm{CDCl}_{3}\right) \delta: 172.36$, $169.96,163.28,138.00,136.75,136.11,135.05,133.62$, $129.47,129.16,128.82,120.08,55.68,52.20,40.35,20.67$,
18.34, 18.06, 14.83; HRMS (ESI) calcd for $\mathrm{C}_{22} \mathrm{H}_{27} \mathrm{~N}_{2} \mathrm{O}_{4}$ $(\mathrm{M}+\mathrm{H})^{+}: 383.1965$, found 383.1970 .

2-[ $N$-(2,6-二甲基苯基)-3-羰基-3-(4-氯苯胺基)丙酰 胺基]丙酸甲酯(7k): 棕色液体, 产率 71\%. ${ }^{1} \mathrm{H}$ NMR (300 $\left.\mathrm{MHz}, \mathrm{CDCl}_{3}\right) \delta: 10.00$ (s, $\left.1 \mathrm{H}, \mathrm{CONH}\right), 7.49 \sim 7.54(\mathrm{~m}, 2 \mathrm{H}$, ArH), $7.12 \sim 7.29(\mathrm{~m}, 5 \mathrm{H}, \operatorname{ArH}), 4.54$ (q, $J=7.4 \mathrm{~Hz}, 1 \mathrm{H}$, $\mathrm{CHCO}$ ), 3.83 (s, $3 \mathrm{H}, \mathrm{COOCH}_{3}$ ), 3.08 (dd, $J=17.9,28.7$ $\mathrm{Hz}, 2 \mathrm{H}, \mathrm{COCH}_{2} \mathrm{CO}$ ), 2.43, 2.14 (2s, 6H, $\left.2 \times \mathrm{ArMe}\right), 1.08$ (d, $\left.J=7.5 \mathrm{~Hz}, 3 \mathrm{H}, \mathrm{CHCH}_{3}\right) ;{ }^{13} \mathrm{C}$ NMR $\left(75 \mathrm{MHz}, \mathrm{CDCl}_{3}\right)$ $\delta: 172.28,169.69,163.71,137.94,136.77,136.33,136.04$, $129.40,129.18,128.80,128.74,128.53,121.05,55.62$, $52.13,40.64,18.32,18.05,14.81$; HRMS (ESI) calcd for $\mathrm{C}_{21} \mathrm{H}_{24} \mathrm{ClN}_{2} \mathrm{O}_{4}(\mathrm{M}+\mathrm{H})^{+}:$403.1419, found 403.1418.

2-[ $\mathrm{N}$-(2,6-二甲基苯基)-3-羰基-3-(5-氟-2-甲基苯胺 基)丙酰胺基]丙酸甲酯(71): 黄色固体, 产率 69\%. m.p. 73 $74{ }^{\circ} \mathrm{C} ;{ }^{1} \mathrm{H}$ NMR $\left(300 \mathrm{MHz}, \mathrm{CDCl}_{3}\right) \delta: 10.02(\mathrm{~s}, 1 \mathrm{H}$, CONH), 7.79 (dd, $J=11.1,2.7 \mathrm{~Hz}, 1 \mathrm{H}, \mathrm{ArH}), 6.96 \sim 7.19$ (m, 4H, ArH), 6.58 $6.64(\mathrm{~m}, 1 \mathrm{H}, \operatorname{ArH}), 4.45$ (q, $J=7.4$ $\mathrm{Hz}, 1 \mathrm{H}, \mathrm{CHCO}$ ), 3.70 (s, $3 \mathrm{H}, \mathrm{COOCH}_{3}$ ), 3.00 (dd, $J=$ 17.6, $\left.24.5 \mathrm{~Hz}, 2 \mathrm{H}, \mathrm{COCH}_{2} \mathrm{CO}\right), 2.34,2,20,2.05(3 \mathrm{~s}, 9 \mathrm{H}$, $3 \times \mathrm{ArMe}), 0.98\left(\mathrm{~d}, J=7.5 \mathrm{~Hz}, 3 \mathrm{H}, \mathrm{CHCH}_{3}\right) ;{ }^{13} \mathrm{C}$ NMR $(75$ $\left.\mathrm{MHz}, \mathrm{CDCl}_{3}\right) \delta: 172.10,169.93,163.41,160.98$ (d, $J=$ $240.1 \mathrm{~Hz}$ ), 137.92, 137.01 (d, $J=11.1 \mathrm{~Hz}), 136.68,135.98$, 130.65 (d, $J=9.0 \mathrm{~Hz}$ ), 129.43, 129.17, 128.79, 122.90 (d, $J=3.2 \mathrm{~Hz}), 110.37$ (d, $J=21.3 \mathrm{~Hz}), 108.31$ (d, $J=26.8$ $\mathrm{Hz}), 55.63,51.97,40.11,18.28,17.93,16.93,14.73$; HRMS (ESI) calcd for $\mathrm{C}_{22} \mathrm{H}_{26} \mathrm{FN}_{2} \mathrm{O}_{4}(\mathrm{M}+\mathrm{H})^{+}:$401.1871, found 401.1874 .

2-[ $N$-(2,6-二甲基苯基)-3-羰基-3-(3-氯-4-甲基苯胺 基)丙酰胺基]丙酸甲酯(7m): 黄色固体，产率 78\%. m.p. $105 \sim 106{ }^{\circ} \mathrm{C} ;{ }^{1} \mathrm{H}$ NMR (300 MHz, $\left.\mathrm{CDCl}_{3}\right) \delta: 9.91(\mathrm{~s}, 1 \mathrm{H}$, CONH), 7.67 (d, $J=2.1 \mathrm{~Hz}, 1 \mathrm{H}, \mathrm{ArH}), 7.30$ (d, $J=2.6 \mathrm{~Hz}$, $1 \mathrm{H}, \operatorname{ArH}), 7.08 \sim 7.27$ (m, 4H, ArH), 4.54 (q, $J=7.4 \mathrm{~Hz}$, $1 \mathrm{H}, \mathrm{CHCO}$ ), $3.81\left(\mathrm{~s}, 3 \mathrm{H}, \mathrm{COOCH}_{3}\right), 3.08$ (dd, $J=17.5$, $\left.29.6 \mathrm{~Hz}, 2 \mathrm{H}, \mathrm{COCH}_{2} \mathrm{CO}\right), 2.43,2$. 29, $2.15(3 \mathrm{~s}, 9 \mathrm{H}$, $3 \times \mathrm{ArMe}), 1.07$ (d, $\left.J=7.5 \mathrm{~Hz}, 3 \mathrm{H}, \mathrm{CHCH}_{3}\right) ;{ }^{13} \mathrm{C} \mathrm{NMR}$ $\left(75 \mathrm{MHz}, \mathrm{CDCl}_{3}\right) \delta: 172.29,169.78,163.54,137.95$, $136.75,136.47,136.04,134.02,131.36,130.59,129.43$, $129.19,128.82,120.39,118.15,55.66,52.17,40.47,19.20$, 18.33, 18.06, 14.81; HRMS (ESI) calcd for $\mathrm{C}_{22} \mathrm{H}_{25} \mathrm{ClN}_{2} \mathrm{O}_{4}$ $(\mathrm{M}+\mathrm{H})^{+}:$417.1576, found 417.1576.

2-[N-(2,6-二甲基苯基)-3-羰基-3-(4-溴-3-三氟甲基 苯胺基)丙酰胺基]丙酸甲酯(7n): 黄色液体, 产率 74\%. ${ }^{1} \mathrm{H}$ NMR (300 MHz, $\mathrm{CDCl}_{3}$ ) $\delta: 10.22$ (s, $1 \mathrm{H}, \mathrm{CONH}$ ), 7.89 (d, $J=2.5 \mathrm{~Hz}, 1 \mathrm{H}, \mathrm{ArH}), 7.66$ (dd, $J=8.7,2.5 \mathrm{~Hz}, 1 \mathrm{H}$, 
ArH), $7.55(\mathrm{~d}, J=8.7 \mathrm{~Hz}, 1 \mathrm{H}, \operatorname{ArH}), 7.12 \sim 7.28(\mathrm{~m}, 3 \mathrm{H}$, $\operatorname{ArH}$ ), 4.55 (q, $J=7.4 \mathrm{~Hz}, 1 \mathrm{H}, \mathrm{CHCO}), 3.81$ (s, 3H, $\left.\mathrm{COOCH}_{3}\right), 3.11\left(\mathrm{dd}, J=17.6,29.5 \mathrm{~Hz}, 2 \mathrm{H}, \mathrm{COCH}_{2} \mathrm{CO}\right)$, 2.44, $2.16(2 \mathrm{~s}, 6 \mathrm{H}, 2 \times \mathrm{ArMe}), 1.07$ (d, $J=7.5 \mathrm{~Hz}, 3 \mathrm{H}$, $\left.\mathrm{CHCH}_{3}\right) ;{ }^{13} \mathrm{C}$ NMR $\left(75 \mathrm{MHz}, \mathrm{CDCl}_{3}\right) \delta: 172.28,169.70$, $164.01,137.92,137.16,136.68,135.90,135.02,129.51$, 129.31, 128.88, 123.79, 119.08 (q, $J=5.7 \mathrm{~Hz}$ ), 55.70, 52.23, 40.46, 18.31, 18.04, 14.78; HRMS (ESI) calcd for $\mathrm{C}_{22} \mathrm{H}_{23} \mathrm{BrF}_{3} \mathrm{~N}_{2} \mathrm{O}_{4}(\mathrm{M}+\mathrm{H})^{+}:$515.0788, found 515.0792.

辅助材料(Supporting Information) 化合物 $\mathbf{2 a} \sim \mathbf{2 n}$, $\mathbf{3 a} \sim 3 \mathbf{n}, \mathbf{7 a} \sim 7 \mathbf{n}$ 的 ${ }^{1} \mathrm{H}$ NMR 谱图和化合物 $\mathbf{2 a} 、 \mathbf{2 b} 、 \mathbf{2 d}$ 、 $2 g 、 2 h 、 2 l 、 2 n 、 3 a 、 3 b 、 3 d 、 3 g 、 3 l 、 3 n, 7 a \sim 7 n$ 的 ${ }^{13} \mathrm{C}$ NMR 谱图和 HRMS. 这些材料可以免费从本刊网站 (http://sioc-journal.cn/)上下载.

\section{References}

[1] Dong, X. X. Med. Philos. 1989, 2, 55 (in Chinese). (董驹翔, 医学与哲学, 1989, 2, 55.)

[2] (a) Liu, C. X.; Wang, R. Z.; Liu, N.; Li, J.; Cheng, X. X. Chin. J. Clin. Pharmacol. Ther. 2004, 9, 56 (in Chinese). (刘昌孝, 王瑞芝, 刘宁, 李晶, 程晓翔, 中国临床药理学与治疗 学, 2004, 9, 56.)

(b) Woods, D D. Br. J. Exp. Pathol. 1940, 21, 74. (c) Fildes P. Lancet 1940, 238, 955.

[3] Lu, Y. L.; Diao, J. L.; Gu, X.; Zhang, Y. F.; Xu, P.; Wang, P.; Zhou, Z. Q. Chirality 2011, 23, 424.

[4] Yaacoby, T.; Hall, J. C.; Stephenson, G. R. Pestic. Biochem. Physiol. 1991, 41, 296.

[5] Karmakar, R.; Ramcharan, B.; Gita, K. J. Agric. Food Chem. 2009, $57,6369$.

[6] Zhang, M. M.; Sun, K.; Wu, H. F.; Yang, H.; Zhang, H.; Zhang, J.; Yang, H. X. Agrochemicals 2010, 49, 94 (in Chinese). (张明媚, 孙克, 吴鸿飞, 杨浩, 张弘, 赵静, 杨贺选, 农药, 2010, 49, 94.)

[7] Chen, Y. W.; Wan, Y. Y.; Liu, Q. X.; Liu, J. B.; Xiong, L. X.; Yu, S. J.; Li, Z. M. Chin. J. Org. Chem. 2014, 34, 882 (in Chinese). (陈有为, 万莹芗, 刘巧霞, 刘敬波, 熊丽霞, 于淑晶, 李正名, 有机化学, 2014, 34, 882.)

[8] Liu, Q. X.; Huang, M. Z.; Liu, A. P.; Nie, S. Q.; Lei, M. X.; Ren , Y. G.; Pei, H.; He, L. Y.; Hu, L.; Hu, A. X. Chin. J. Org. Chem. 2014, 34, 118 (in Chinese). (刘祈星, 黄明智, 柳爱平, 聂思桥, 雷满香, 任叶果, 裴晖, 何 丽英，胡礼，胡艾希，有机化学, 2014, 34, 118.)

[9] Xie, F.; Liu, T. T.; Yang, G.; Yuan, J.; Kong, X. L.; Xu, T. M.; Tan, C. X. Chin. J. Org. Chem. 2013, 33, 2596 (in Chinese). (谢峰, 刘婷婷, 杨果, 袁 静, 孔小林, 许天明, 谭成侠, 有机化 学, 2013, 33, 2596.)

[10] Ning, L. H.; Peng, H.; Xu, H. Y.; He, H. W. Chin. J. Pestic. Sci. 2004, 6, 74 (in Chinese). (宁丽红, 彭浩, 涂海洋, 贺红武, 农药学学报, 2004, 6, 74.)

[11] Liu, T. T.; Nie, Y.; Zhong, L. K.; Huang, H. Y.; Hu. W. Q.; Xu, T. M.; Tan, C. X. Chin. J. Org. Chem. 2015, 35, 422 (in Chinese). (刘婷婷, 倪芸, 钟良坤, 黄红英, 胡伟群, 许天明, 谭成侠, 有 机化学, 2015, 35, 422.)

[12] Chen, Z. X.; Wei, M. X.; Li, X. Q.; Li, T. C.; Zhou, X. Z. Chin. J.
Org. Chem. 2015, 35, 835 (in Chinese)

(陈凑喜, 魏梦雪, 李学强, 李天才, 周学章, 有机化学, 2015, 35, 835.)

[13] Zhang, D. Q.; Xu, G. F.; Liu, Y. H.; Wang, D. Q.; Yang, X. L.; Yuan, D. K. Chin. J. Org. Chem. 2015, 35, 2191 (in Chinese). (张大强, 徐高飞, 刘艳红, 王道全, 杨新玲, 袁德凯, 有机化学, 2015, 35, 2191.)

[14] Nie, Y.; Xu, T. M.; Zhong, L. K.; Kong, X. Y.; Shi, J. J.; Liu, X. H.; Kong, X. L.; Ji, W. J.; Tan, C. X. Chin. J. Org. Chem. 2015, 35, 2218 (in Chinese)

(倪芸，许天明，钟良坤，孔晓燕，史建俊，刘幸海，孔小林，姬 文娟, 谭成侠, 有机化学, 2015, 35, 2218.)

[15] Zhang, H.; Liu, R. Q.; Li, Q. B.; Li, Q. Y.; Chen, Y. H.; Liu, K. C.; Liu, S. Z. Chin. J. Org. Chem. 2015, 35, 1506 (in Chinese).

(张浩, 刘瑞全, 李奇博, 李青阳, 陈亚红, 刘克昌, 刘尚钟, 有 机化学, 2015, 35, 1506.)

[16] Li, X. S. Hunan Chem. 1994, (3), 31 (in Chinese). (李湘生, 湖南化工, 1994, (3), 31.)

[17] Gao, Y. X.; Chen, J. H.; Liu, C.; Lv, X. T.; Li, J. Z.; Guo, B. Y. J. Agric. Food Chem. 2013, 61, 9045

[18] Paolo, C.; Marco, M.; Filippo, M. P. J. Chromatogr. 1987, 391, 334.

[19] Liu, M. K.; Liu, D. H.; Xu, Y. G.; Jing, X.; Zhou, Z. Q.; Wang, P. J. Agric. Food Chem. 2015, 61, 5205.

[20] Zhang, X.; Zhang, Y. J.; Chen, Y.; Zhou, M. G. Chin. J. Pestic. Sci. 2008, 10, 41 (in Chinese).

(张晓，张艳军，陈雨，周明国，农药学学报, 2008, 10, 41.)

[21] Freeman, G. A.; Andrews III, C. W.; Hopkins, A. L.; Lowell, G. S.; Schaller, L. T.; Cowan, J. R.; Gonzales, S. S.; Koszalka, G. W.; Hazen, R. J.; Boone, L. R.; Ferris, R. G.; Creech, K. L.; Roberts, G. B.; Short, S. A.; Weaver, K.; Reynolds, D. J.; Milton, J.; Ren, J. S.; Stuart, D. I.; Stammers, D. K.; Chan, J. H. J. Med. Chem. 2004, 47, 5923.

[22] Noboru, S.; Toshio, U. Bull. Fac. Agric., Meiji Univ. 1986, 47.

[23] Renhowe, P. A.; Pecchi, S.; Shafer, C. M.; Machajewski, T. D.; Jazan, E. M.; Taylor, C.; Antonios-McCrea, W.; McBride, C. M.; Frazier, K.; Wiesmann, M.; Lapointe, G. R.; Feucht, P. H.; Warne, R. L.; Heise, C. C.; Menezes, D.; Aardalen, K.; Ye, H. L.; He, M.; Le, V.; Vora, J.; Jansen, J. M.; Wernette-Hammond, M. E.; Harris, A. L. J. Med. Chem. 2009, 52, 278.

[24] Tamura, H.; Matsuda, T.; Komiyama, T. JP 51126127, 1976 [Chem. Abstr. 1977, 87, 9352].

[25] Goodfellow, V. S.; Settineri, M.; Lawton, R. G. Biochemistry 1989, $28,6346$.

[26] Ramana Reddy, M. V.; Akula, B.; Cosenza, S. C.; Athuluridivakar, S.; Mallireddigari, M. R.; Pallela, V. R.; Billa, V. K.; Subbaiah, D. R. C. V.; Bharathi, E. V.; Vasquez-Del Carpio, R.; Padgaonkar, A.; Baker, S. J.; Reddy, E. P. J. Med. Chem. 2014, 57, 578.

[27] Bruge, D.; Buchstaller, H. P.; Wiesner, M.; Finsinger, D.; Baumgarth, M.; Sirrenberg, C.; Zenke, F.; Amendt, C.; Grell, M. WO 2005005389, 2005 [Chem. Abstr. 2005, 142, 134581].

[28] Anandan, S. K.; Ward, J. S.; Brokx, R. D.; Bray, M. R.; Patel, D. V.; Xiao, X. X. Bioorg. Med. Chem. Lett. 2005, 15, 1969.

[29] Pradhan, A.; Pradhan, V.; Jolly, V. S. Orient. J. Chem. 1994, 10, 73.

[30] Veronese, A. C.; Callegari, R.; Morelli, C. F. Tetrahedron 1995, 51, 12277.

[31] Pareek, A. K.; Joseph, P. E.; SethbDaya, S. Orient. J. Chem. 2009, 25, 681 .

[32] Fache, F.; Valot, F.; Milenkovic, A.; Lemaire, M. Tetrahedron 1996, 52, 9777.

[33] Zheng, L. Y.; Zhang, S. Q.; Zhao, L. F.; Zhu, G. S.; Yang, X. Y.; Gao, G.; Cao, S. G. J. Mol. Catal. B: Enzym. 2006, 38, 119.

(Lu, Y.) 